ARTICLE

\title{
An L-2-hydroxyglutarate biosensor based on specific transcriptional regulator LhgR
}

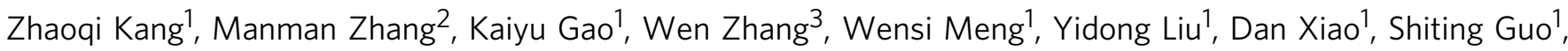
Cuiqing Ma', Chao Gao (i) ${ }^{1 凶} \&$ Ping Xu (i) ${ }^{4 凶}$

L-2-Hydroxyglutarate ( $\mathrm{L}-2-\mathrm{HG}$ ) plays important roles in diverse physiological processes, such as carbon starvation response, tumorigenesis, and hypoxic adaptation. Despite its importance and intensively studied metabolism, regulation of L-2-HG metabolism remains poorly understood and none of regulator specifically responded to L-2-HG has been identified. Based on bacterial genomic neighborhood analysis of the gene encoding L-2-HG oxidase (LhgO), LhgR, which represses the transcription of IhgO in Pseudomonas putida W619, is identified in this study. LhgR is demonstrated to recognize L-2-HG as its specific effector molecule, and this allosteric transcription factor is then used as a biorecognition element to construct an L-2-HG-sensing FRET sensor. The L-2-HG sensor is able to conveniently monitor the concentrations of L-2-HG in various biological samples. In addition to bacterial L-2-HG generation during carbon starvation, biological function of the $L-2-H G$ dehydrogenase and hypoxia induced L-2-HG accumulation are also revealed by using the L-2-HG sensor in human cells.

\footnotetext{
${ }^{1}$ State Key Laboratory of Microbial Technology, Shandong University, Qingdao, People's Republic of China. ${ }^{2}$ Tianjin Key Laboratory of Radiation Medicine and Molecular Nuclear Medicine, Department of Radiobiology, Institute of Radiation Medicine of Chinese Academy of Medical Science and Peking Union Medical College, Tianjin, People's Republic of China. ${ }^{3}$ Center for Gene and Immunotherapy, The Second Hospital of Shandong University, Jinan, People's Republic of China. ${ }^{4}$ State Key Laboratory of Microbial Metabolism, Joint International Research Laboratory of Metabolic and Developmental Sciences, and School of Life Sciences and Biotechnology, Shanghai Jiao Tong University, Shanghai, People's Republic of China. ${ }^{\bowtie}$ email: jieerbu@sdu.edu.cn; pingxu@sjtu.edu.cn
} 
-2-Hydroxyglutarate (L-2-HG) is an important metabolite in various domains of life. In mammals and plants, it is produced by lactate dehydrogenase $(\mathrm{LDH})$ and malate dehydrogenase (MDH)-mediated 2-ketoglutarate (2-KG) reduction under hypoxic conditions ${ }^{1-5}$. In microorganisms, it is a metabolic intermediate of glutarate catabolism produced by a glutarate hydroxylase, $\mathrm{CsiD}^{6-8}$. L-2-HG dehydrogenase (L2HGDH) or L-2-HG oxidase (LhgO), an FAD-containing oxidoreductase that converts $\mathrm{L}-2-\mathrm{HG}$ to $2-\mathrm{KG}$, plays an indispensable role in the catabolism of $\mathrm{L}-2-\mathrm{HG}^{7,9}$. While extensive efforts have been devoted to investigating L-2-HG anabolism and catabolism, the molecular machinery that specifically senses L-2-HG and regulates its metabolism has not been identified until now.

$\mathrm{L}-2-\mathrm{HG}$ is an inhibitor of $2-\mathrm{KG}$ dependent dioxygenases with specific pro-oncogenic capabilities ${ }^{10,11}$. Thus, this oncometabolite is viewed as a biomarker for a variety of cancers and its rapid and sensitive measurement in body fluids is of clinical significance $^{12-15}$. Importantly, L-2-HG also has endogenous functions in healthy animal cells. For example, this compound was recently identified to aid the proliferation and antitumorigenic abilities of $\mathrm{CD} 8^{+} \mathrm{T}$-lymphocytes ${ }^{16}$, to contribute to relieving the cellular reductive stress ${ }^{3}$, and to coordinate glycolytic flux with epigenetic modifications ${ }^{17}$. Considering the various roles of L-2-HG in cell metabolism, the development and optimization of real-time monitoring assays for this metabolite in living cells are required.

Liquid chromatography-tandem mass spectrometry (LC-MS/ MS) ${ }^{18,19}$ and gas chromatography-tandem mass spectrometry $(\mathrm{GC}-\mathrm{MS} / \mathrm{MS})^{20,21}$ are often used to assess the extracellular concentrations of L-2-HG. These state-of-the-art methods are timeconsuming, expensive to perform, and require highly skilled personnel. In addition, these destructive methods are also incompatible with real-time monitoring of the fluctuations of L-2HG concentrations in intact living cells. In this study, we identify and characterize LhgR, an L-2-HG catabolism regulator in Pseudomonas putida W619. Mechanistically, LhgR represses the transcription of LhgO encoding gene $\operatorname{lhgO}$. L-2-HG is a specific effector molecule of LhgR and prevents LhgR binding to the promoter region of $l h g O$. We then report the development and application of the LhgR-based L-2-HG biosensor via Förster resonance energy transfer (FRET), a technology widely applied to investigate temporal dynamics of various small molecules, such as potassium $^{22,23}$, glycine ${ }^{24}$, and cAMP $^{25,26}$. As-designed sensor quantitatively responds to L-2-HG concentrations in various biological samples with high accuracy and precision. We also use this biosensor to identify the carbon starvation-induced L-2-HG production in bacteria and to demonstrate hypoxia-induced L-2HG production by $\mathrm{LDH}$ and $\mathrm{MDH}$ in human cells. Therefore, the LhgR-based biosensor can prove to be a useful tool for real-time measurement of the L-2-HG concentrations in living cells.

\section{Results}

LhgR regulates L-2-HG catabolism. In this study, bacteria containing LhgO encoding gene lhgO were selected to study the regulation of L-2-HG metabolism. Homologs of LhgO can be found in 612 different bacterial strains. Similarly organized chromosomal clusters are found in many bacterial genomes, which contain various combinations of genes related to glutarate metabolism (csiD, lhgO, gabT, gabD, and gabP) (Fig. 1a). In Pseudomonas putida KT2440, the glutarate regulon is regulated by allosteric transcription factor CsiR, which is encoded upstream of $c s i D^{27}$. The glutarate sensing allosteric transcription factor CsiR and its cognate promoter were cloned into broad host range vectors to create a glutarate biosensor ${ }^{28}$. Interestingly, a different pattern of $l$ ggO gene neighborhood was observed in a few species that do not contain $c s i D$ homologs (Fig. 1a). For example, a gene encoding a GntR family protein, $l h g R$, was found directly upstream of $l$ hgO in P. putida W619. The absence of csiD gene related to glutarate catabolism made us to reason that $\operatorname{lhgO}$ of P. putida W619 might be solely involved in L-2-HG metabolism and be $\mathrm{L}-2-\mathrm{HG}$ inducible.

The lhgO gene in P. putida W619 was cloned into pME6032 vector and transferred into $P$. putida KT2440 ( $\Delta l h g O)$. As shown in Fig. 1b, c, the complement of lhgO in P. putida W619 could restore glutarate and L-2-HG utilization abilities of $P$. putida $\mathrm{KT} 2440(\Delta l h g O)$, confirming that $l h g O$ encodes a functional L-2HG catabolic enzyme. To identify the function of LhgR in $P$. putida W619, the gene segment F2-lhgR-F1-lhgO, which contains the promoter of $\operatorname{lh} g R(\mathrm{~F} 2), \operatorname{lng} R$, the promoter of $\operatorname{lhgO}(\mathrm{F} 1)$, and lhgO, was cloned into pME6032 vector, and the resulting plasmid was transferred into different derivatives of $P$. putida KT2440 (Fig. 1d). As shown in Fig. 1e, exogenous L-2-HG, but not its mirror-image enantiomer $\mathrm{D}-2-\mathrm{HG}$, can induce the expression of $\operatorname{lhgO}$ in the gene segment F2-lhgR-F1-lhgO and restore LhgO activity in $P$. putida KT2440 ( $\Delta c s i R \Delta l h g O)$. In addition, the activity of LhgO was also detected in $P$. putida KT2440 $(\Delta c s i R \Delta l h g O)$ harboring pME6032-F2-lhgR-F1-lhgO when cultured with glutarate as the sole carbon source. However, no activity of LhgO was detected in $P$. putida KT2440 ( $\Delta c s i R \Delta c$ siD $\operatorname{lihgO}$ ), in which the key gene responsible for L-2-HG production from glutarate was deleted. These results indicated that LhgR represses the expression of LhgO and L-2-HG, but neither $\mathrm{D}-2-\mathrm{HG}$ nor glutarate can serve as the effector molecule of LhgR.

LhgR specifically responds to L-2-HG. To determine whether LhgR directly interacts with the promoter region of $\operatorname{lhgO}$, LhgR in P. putida W619 was overexpressed in E. coli BL21(DE3) and purified by Ni-chelating chromatography (Fig. 2a). Based on the results of gel filtration and sodium dodecyl sulfatepolyacrylamide gel electrophoresis (SDS-PAGE), LhgR behaved as a dimer (Fig. 2b). Subsequently, electrophoretic mobility shift assays (EMSAs) were conducted using lhgO promoter (F1) and purified LhgR. As shown in Fig. 2c, LhgR bound to F1 in a concentration-dependent manner. LhgR completely shifted fragment F1 gel band when an 8-fold molar excess was used. A DNase I footprinting assay was also performed using purified LhgR and fragment F1. A protected region containing palindromic $\mathrm{N}_{\mathrm{y}} \mathrm{GTN}_{\mathrm{x}} \mathbf{A C N}_{\mathrm{y}}$ consensus binding motif of GntR-family allosteric transcription factor ${ }^{29}$, 5'-TAGTCTGACAA-3', was observed (Fig. 2d). In addition, LhgR also bound to its promoter (F2) in EMSAs. A similar consensus binding motif, 5'TTGTCTGACAA-3', was protected in DNase I footprinting assay (Supplementary Fig. 1a-b).

Effects of L-2-HG, D-2-HG, glutarate, 2-KG, L-lysine, 5aminovalerate, and succinate on LhgR binding to the $\operatorname{lhg} O$ promoter region F1 were also assessed by EMSAs. The release of LhgR from fragments F1 was observed only in the presence of L-2-HG (Fig. 2e). These results suggested that $\mathrm{L}-2-\mathrm{HG}$ can specifically prevent the binding of LhgR to the promoter of $\operatorname{lngO}$ and induce its expression. LhgR may help P. putida W619 to specifically sense L-2-HG generated by intracellular metabolism or present in habitats and stimulate the catabolism of L-2-HG. In addition, LhgR may self-repress its expression and L-2-HG can also contribute to inducing the expression of $\operatorname{lhg} R$ (Supplementary Fig. 1c).

Design and optimization of the L-2-HG-sensing reporter. FRET sensors, which combine a ligand-binding moiety and a pair of donor-acceptor fluorescent pair, allow measurement of ligand concentrations based on the ligand-binding induced changes of 
a

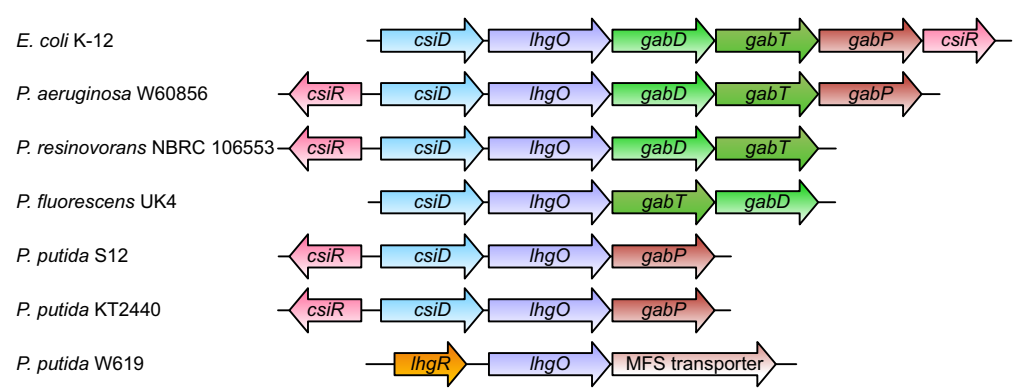

b
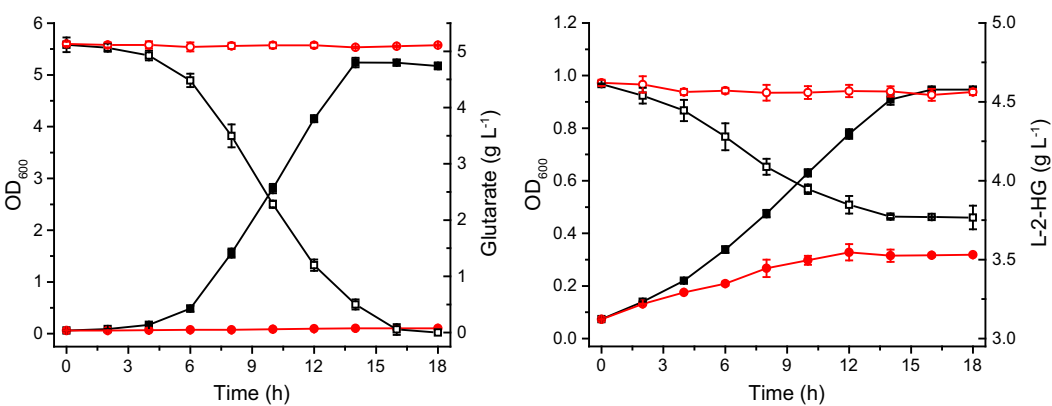

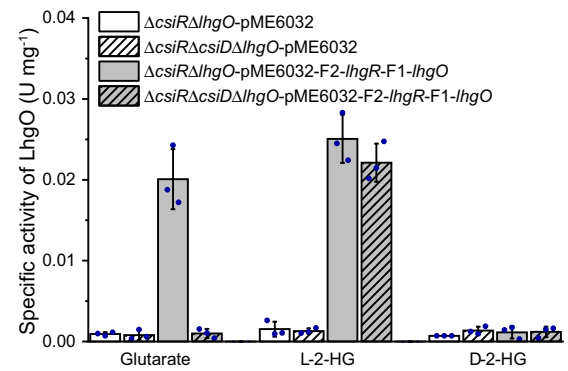

d

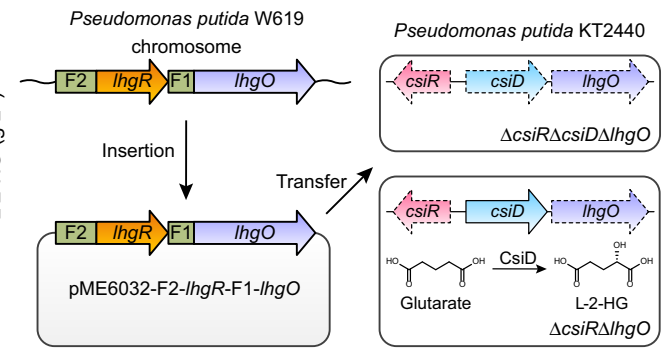

Fig. 1 Regulation of L-2-HG catabolism by LhgR in P. putida W619. a Schematic representation of genomic neighborhood analysis of IhgO in different bacteria. Orthologs are shown in the same color and the direction of gene transcription is indicated by arrows. CsiR, GntR family allosteric transcription factor regulating glutarate catabolism; CsiD, glutarate hydroxylase; LhgO, L-2-HG oxidase; GabD, succinate semialdehyde dehydrogenase; GabT, 4aminobutyrate aminotransferase; GabP, 4-aminobutyrate transporter. b, c Growth of the derivatives of $P$. putida KT2440 in MSMs with glutarate (b) or L-2$\mathrm{HG}$ (c) as the sole carbon source. Growth (closed symbols) and the consumption of carbon source (open symbols) of $P$. putida KT2440 ( $\Delta$ /hgO) harboring plasmid pME6032-IhgO (black lines with squares) and P. putida KT2440 ( $\Delta / \mathrm{hgO}$ ) harboring empty plasmid pME6032 (red lines with circles) were measured in MSMs containing $5 \mathrm{~g} \mathrm{~L}^{-1}$ glutarate (b) or L-2-HG (c) as the sole carbon source. d Schematic representation of the construction of pME6032F2-IhgR-F1-IhgO and its transfer into P. putida KT2440 ( $\Delta c s i R \Delta / h g O)$ and P. putida KT2440 ( $\Delta c s i R \Delta c s i D \Delta / h g O)$ by electroporation. The deleted genes in $P$. putida KT2440 are indicated by dashed lines. The reaction catalyzed by CsiD is also demonstrated. e The activities of LhgO in $P$. putida KT2440 $(\Delta c s i R \Delta / h g O)$ and $P$. putida KT2440 ( $\Delta c s i R \Delta c s i D \Delta / h g O)$ harboring either plasmid pME6032-F2-IhgR-F1-IhgO or empty plasmid pME6032 grown in MSM with glutarate, $\mathrm{L}-2-\mathrm{HG}$, or $\mathrm{D}-2-\mathrm{HG}$ as the sole carbon source. All data shown are means \pm standard deviations (s.d.) $(n=3$ independent experiments).

FRET efficiency ${ }^{22-26}$. In this study, the L-2-HG-sensing fluorescent reporter (LHGFR) was constructed by fusion of the optimized cyan and yellow fluorescent protein variants, TFPP $^{30}$ and Venus $^{31}$, to the N-terminus and C-terminus of LhgR (Supplementary Fig. 2). This first LHGFR was named LHGFR $_{0 \mathrm{NOC}}$, where the subscript indicates the number of amino acids truncated from the N-terminus and C-terminus of LhgR. Subsequently, LHGFR $_{0 \mathrm{NOC}}$ was overexpressed in E. coli BL21(DE3) and purified by a Ni-chelating chromatographic column (Supplementary Fig. 3). Spectra properties of $\mathrm{LHGFR}_{0 \mathrm{NOC}}$ reveal the addition of $\mathrm{L}$ 2-HG could reduce the emission peak at $492 \mathrm{~nm}$ of mTFP and increase the emission peak at $526 \mathrm{~nm}$ of Venus (Supplementary Fig. 4). Thus, the conformational change of LhgR after the L-2HG binding may lead to a shortened relative distance and/or favorable orientation of mTFP and Venus, resulting in an increase in FRET (Fig. 3a). In addition, L-2-HG increased the emission ratio of Venus to $\mathrm{mTFP}$ in a dose-dependent manner, with a maximum ratio change $\left(\Delta R_{\max }\right)$ of $11.47 \pm 0.38 \%$, an apparent dissociation constant $\left(K_{d}\right)$ of $2.74 \pm 0.73 \mu \mathrm{M}$, and a Hill slope close to 1 (Fig. 3b).

To increase the magnitude of responses, LHGFR was optimized by truncating $\mathrm{N}$-terminal and C-terminal amino acids of LhgR or by adding a series of artificial linkers between LhgR and various fluorescent proteins ${ }^{23,32-34}$ (Fig. 3c and Supplementary Fig. 5). Truncation of three to seven C-terminal amino acids in LhgR could significantly increase $\Delta R_{\max }$ of the sensor (Fig. $3 \mathrm{c}$, $\mathrm{d}$ and Supplementary Fig. 5). Among the five sensors with increased response magnitude values, LHGFR $_{0 \text { N7C }}$ exhibited the highest $\Delta R_{\max }$ of $60.37 \pm 1.30 \%$ and $K_{d}$ of $7.22 \pm 0.38 \mu \mathrm{M}$ (Fig. $3 \mathrm{~d}$ and
Supplementary Fig. 6a-e). In addition, LHGFR $_{0 \mathrm{~N} 3 \mathrm{C}}$ was also a promising sensor with a high $\Delta R_{\max }$ of $56.13 \pm 0.29 \%$ and a high $K_{d}$ of $29.33 \pm 1.24 \mu \mathrm{M}$ (Fig. 3d and Supplementary Fig. 6a).

Then, the properties of $\mathrm{LHGFR}_{0 \mathrm{~N} 3 \mathrm{C}}$ and $\mathrm{LHGFR}_{0 \mathrm{~N} 7 \mathrm{C}}$ were also investigated. Both $\mathrm{LHGFR}_{0 \mathrm{~N} 3 \mathrm{C}}$ and $\mathrm{LHGFR}_{0 \mathrm{~N} 7 \mathrm{C}}$ behave as tetramers and have lost the ability to bind DNA (Supplementary Fig. 7). L-2-HG binding increased FRET between the fluorophores in LHGFR $_{0 \mathrm{~N} 3 \mathrm{C}}$ and $\mathrm{LHGFR}_{0 \mathrm{~N} 7 \mathrm{C}}$ (Supplementary Fig. 8). DLactate, L-lactate, as well as a set of intermediates of TCA cycle and L-lysine catabolism, were used to examine the specificity of LHGFR $_{0 \mathrm{~N} 3 \mathrm{C}}$ and $\mathrm{LHGFR}_{0 \mathrm{~N} 7 \mathrm{C}}$. None of D-lactate, L-lactate, oxaloacetate, citrate, isocitrate, $2-\mathrm{KG}$, succinate, fumarate, cisaconitate, L-malate, pyruvate, L-lysine, 5-aminovalerate, and glutarate induced the emission ratio changes of $\mathrm{LHGFR}_{0 \mathrm{~N} 3 \mathrm{C}}$ or LHGFR $_{0 \mathrm{N7C}}$ (Fig. 3e, f). The addition of these compounds also had no influence on the response of $\mathrm{LHGFR}_{0 \mathrm{~N} 3 \mathrm{C}}$ or $\mathrm{LHGFR}_{0 \mathrm{~N} 7 \mathrm{C}}$ to L-2-HG (Supplementary Fig. 9a-b). Both LHGFR $_{0 \mathrm{~N} 3 \mathrm{C}}$ and LHGFR $_{0 \text { N7C }}$ exhibited much higher affinity for L-2-HG than that for D-2-HG (Supplementary Fig. 9c-d and Supplementary Table 1). The limits of detection (LODs) of $\mathrm{LHGFR}_{0 \mathrm{~N} 3 \mathrm{C}}$ and LHGFR $_{\text {ON7C }}$ for L-2-HG and D-2-HG were $4.34 \mu \mathrm{M}$ and 872.59 $\mu \mathrm{M}, \quad 0.70 \mu \mathrm{M}$ and $128.34 \mu \mathrm{M}$, respectively (Supplementary Table 1). The dose-response curves of LHGFR $_{0 \mathrm{~N} 3 \mathrm{C}}$ and LHGFR $_{0 \mathrm{~N} 7 \mathrm{C}}$ for L-2-HG in the absence or presence of $\mathrm{D}-2-\mathrm{HG}$, 2-KG, and ATP were also assayed (Supplementary Fig. 9e-j). Similar LODs, $K_{d}$ values, and $\Delta R_{\max }$ of LHGFR 0 N3C were detected (Supplementary Fig. 9e-j and Supplementary Table 1). Kinetic analyses of $\mathrm{LHGFR}_{0 \mathrm{~N} 3 \mathrm{C}}$ and $\mathrm{LHGFR}_{0 \mathrm{~N} 7 \mathrm{C}}$ were performed and values for the association rate constant $\left(k_{\mathrm{on}}\right)$ and dissociation rate 
a

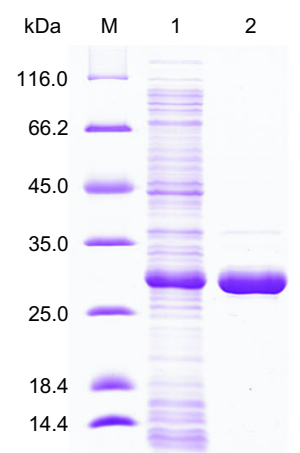

b

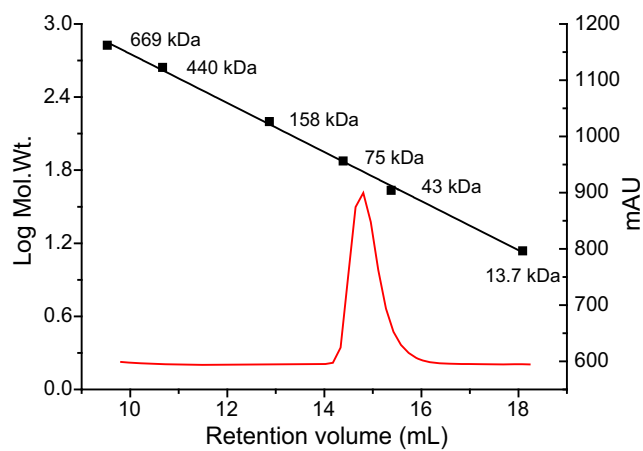

c

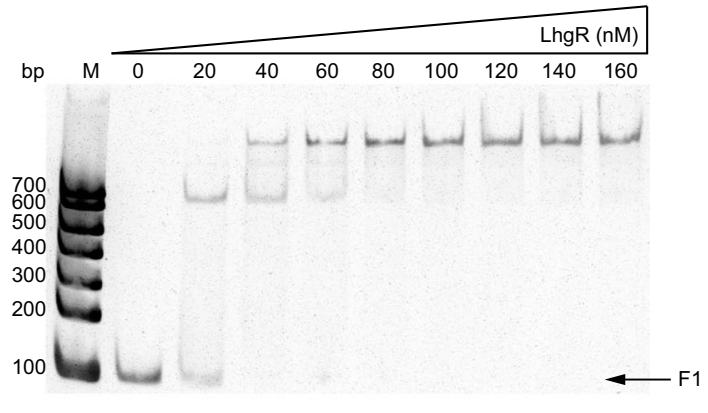

d

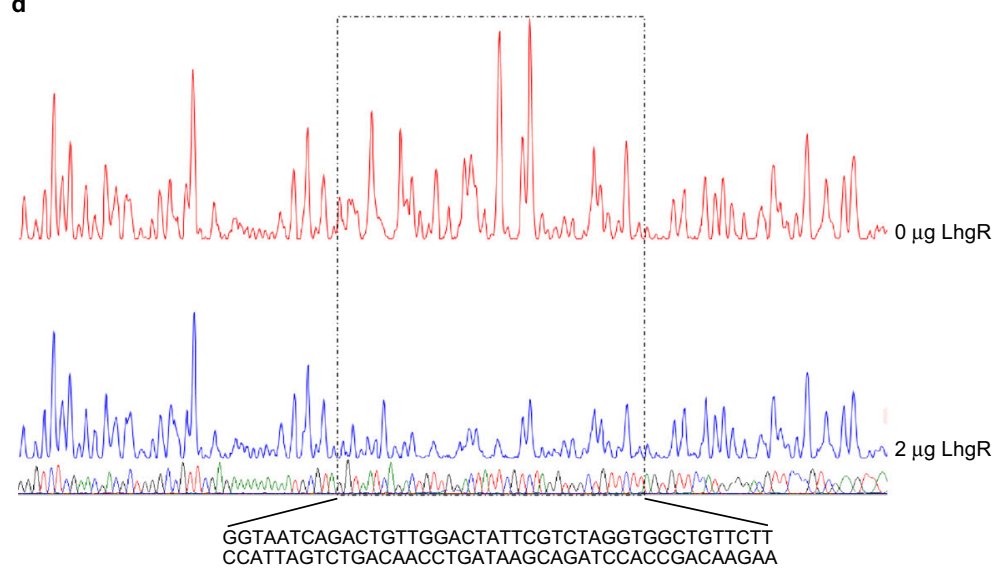

e

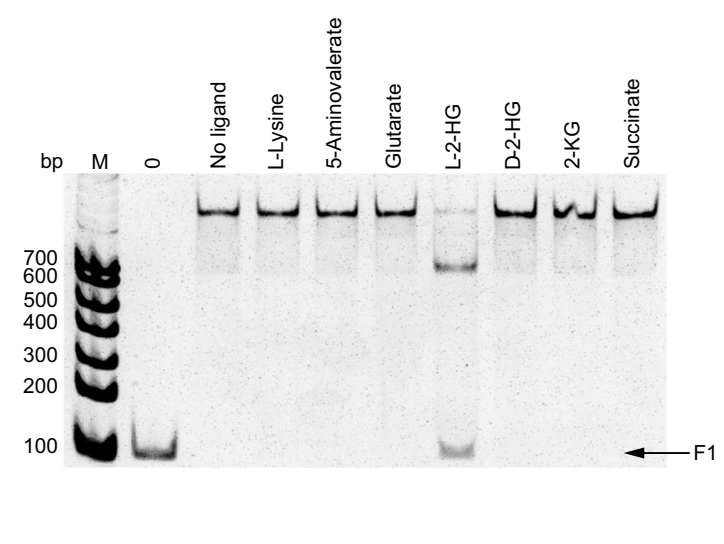

Fig. 2 Purification and characterization of LhgR. a SDS-PAGE analysis of the purification of LhgR. Lane M, molecular weight markers; lane 1, crude extract of E. coli BL21(DE3) harboring pETDuet-IhgR; lane 2, purified His ${ }_{6}$-tagged LhgR using a HisTrap column. b Gel-filtration chromatography of the purified LhgR with the Superdex 200 10/300 GL column. Red curve, the chromatogram of purified LhgR; Black line, a standard curve for protein molecular mass standards. $\mathbf{c} \mathrm{LhgR}$ can bind to the IhgO promoter region. F1 fragment containing the IhgO promoter region (10 nM) was titrated by purified LhgR ( 0,20 , 40, $60,80,100,120,140,160 \mathrm{nM}$ ). Lane $\mathrm{M}$, molecular weight markers. $\mathbf{d}$ DNase I footprinting analysis of LhgR binding to the IhgO promoter region. The F1 fragment was labeled with 6-carboxyfluorescein (FAM) and incubated with $2 \mu \mathrm{L}$ LhgR (blue line) or without LhgR (red line). The region protected by LhgR is indicated with a dotted box. e L-2-HG prevents LhgR binding to the IhgO promoter region. EMSAs were carried out with F1 fragment (10 nM) and purified LhgR $(60 \mathrm{nM})$ in the absence of any other tested compounds (No ligand) and in the presence of $50 \mathrm{mM}$ different compounds. Lane $\mathrm{M}$ was the molecular weight marker; lane 0 without LhgR was used as the control.

constant $\left(k_{\text {off }}\right)$ of LHGFR LN3C $_{\text {and LHGFR }}$ aN7C were determined to be $5.50 \times 10^{-1} \mu \mathrm{M}^{-1} \mathrm{~s}^{-1}$ and $15.75 \mathrm{~s}^{-1}, 2.84 \times 10^{-1} \mu \mathrm{M}^{-1} \mathrm{~s}^{-1}$ and $5.79 \mathrm{~s}^{-1}$, respectively (Supplementary Fig. 10a-d). Effects of temperature on $\mathrm{LHGFR}_{0 \mathrm{~N} 3 \mathrm{C}}$ and $\mathrm{LHGFR}_{0 \mathrm{~N} 7 \mathrm{C}}$ were analyzed, respectively, and the affinity of $\mathrm{LHGFR}_{0 \mathrm{~N} 3 \mathrm{C}}$ to $\mathrm{L}-2-\mathrm{HG}$ remained unaffected from $25^{\circ} \mathrm{C}$ to $37^{\circ} \mathrm{C}$ (Supplementary Fig. 10e-h). L-2-HG-dependent emission ratio changes of LHGFR $_{0 \mathrm{~N} 3 \mathrm{C}}$ or LHGFR $_{\text {ON7C }}$ were reversible by L-2-HG oxidation catalyzed by 5 $\mu \mathrm{M}$ LhgO (Fig. 3g, h and Supplementary Fig. 10i-j) and both biosensors were stable for the detection of L-2-HG from $\mathrm{pH} 6.0$ to 8.0 (Supplementary Fig. 10k-1).

Characterization of LHGFR in biological samples. Next, we investigated whether LHGFR could be used to quantify L-2-HG concentrations in different biological samples. When L-2-HG with increasing concentrations (0 to $2 \mathrm{mM}$ ) were added into serum and urine samples of healthy adults, the response curves were nearly identical with that in assay buffer for both LHGFR $_{0 \mathrm{~N} 3 \mathrm{C}}$ and $\mathrm{LHGFR}_{0 \mathrm{~N} 7 \mathrm{C}}$ (Fig. 4a-d, Supplementary Fig. 6a and Fig. 6e). Thus, quantitative determination of L-2-HG could be conducted by mixing the target samples with LHGFR and measuring the emission ratios with a conventional fluorescence microplate reader. Based on the response curves established for L-2-HG quantification, both biosensors were used to assay the concentrations of L-2-HG in human serum and urine (Fig. 4e, f).
The results of $\mathrm{LHGFR}_{0 \mathrm{~N} 3 \mathrm{C}}$ and $\mathrm{LHGFR}_{0 \mathrm{~N} 7 \mathrm{C}}$ showed close agreement with the results of LC-MS/MS, the current standard method for clinical assays of L-2-HG (Fig. 4e, f and Supplementary Table 2).

In a previous report, $\mathrm{L}-2-\mathrm{HG}$ was identified to be a metabolic intermediate of glutarate metabolism in $P$. putida $\mathrm{KT}_{2} 440^{7}$. LHGFR $_{0 \mathrm{~N} 3 \mathrm{C}}$ and $\mathrm{LHGFR}_{0 \mathrm{~N} 7 \mathrm{C}}$ also exhibited high accuracy in the quantification of L-2-HG in bacterial culture medium (Fig. 4g-i). When cultured in the medium containing $20 \mathrm{mM}$ glucose and 5 $\mathrm{mM}$ glutarate as carbon sources, the growth of $P$. putida KT2440 $(\Delta l h g O)$ was significantly delayed, which might be due to the possible toxicity of accumulated L-2-HG (Fig. 4j). Nearly identical results of L-2-HG quantification were also obtained by either using $\mathrm{LHGFR}_{0 \mathrm{~N} 3 \mathrm{C}}, \mathrm{LHGFR}_{0 \mathrm{~N} 7 \mathrm{C}}$, or using LC-MS/MS (Fig. 4k, 1 and Supplementary Fig. 11). Mutual corroboration between the two biosensors further confirmed their applicability in in vitro L-2-HG quantification of various biological samples. In addition, the response curves of $\mathrm{LHGFR}_{0 \mathrm{~N} 3 \mathrm{C}}$ and $\mathrm{LHGFR}_{0 \mathrm{~N} 7 \mathrm{C}}$ for $\mathrm{D}-2-\mathrm{HG}$ in serum, urine, and bacterial culture medium were also determined (Supplementary Fig. 12). The LODs of $\mathrm{LHGFR}_{0 \mathrm{~N} 3 \mathrm{C}}$ and LHGFR $_{0 \mathrm{~N} 7 \mathrm{C}}$ for $\mathrm{D}-2-\mathrm{HG}$ were more than 100-fold higher than those for L-2-HG (Supplementary Table 3 and Table 4).

Monitoring L-2-HG fluctuations in living bacteria by LHGFR. We also investigated whether the LhgR-based L-2-HG sensor 
a

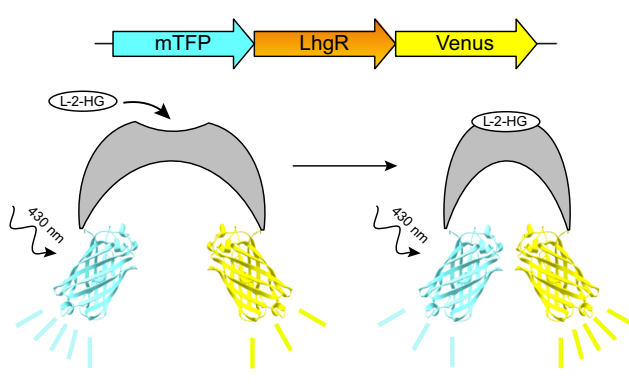

d

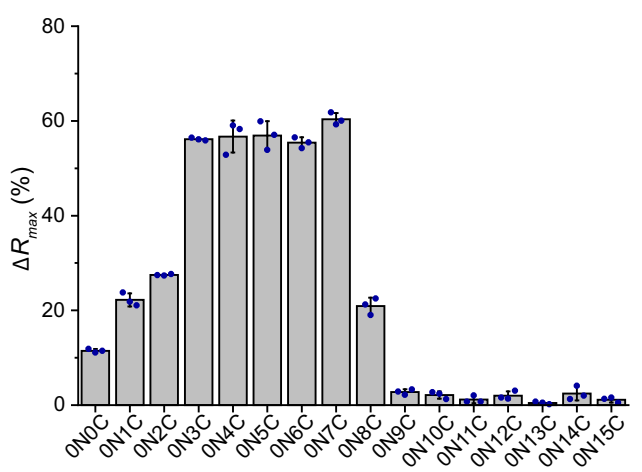

$\mathbf{f}$

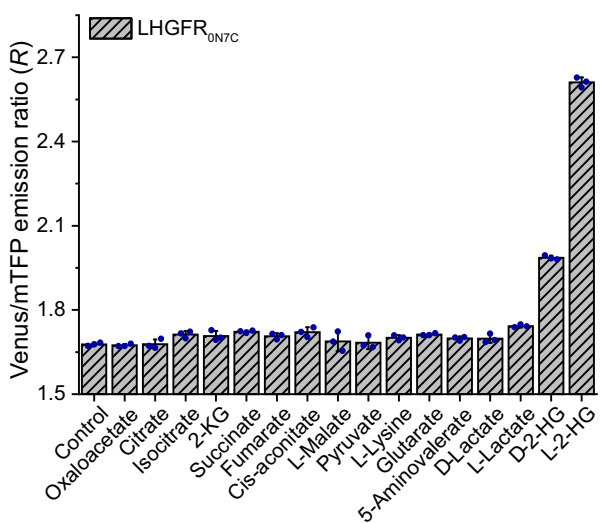

b
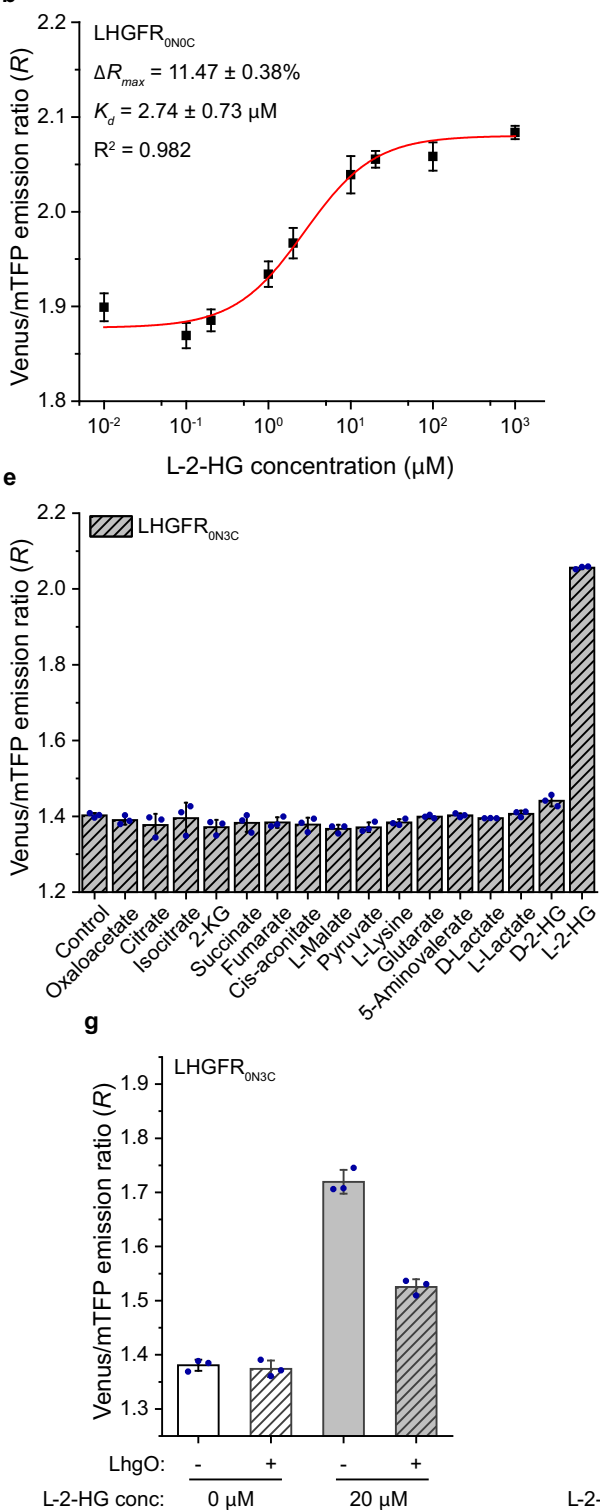

C

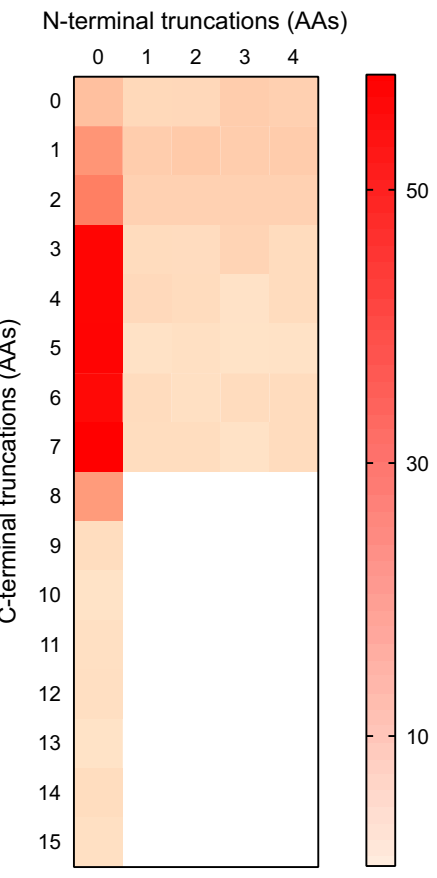

Fig. 3 Design and optimization of the LHGFR. a Schematic representation of the predicted conformational change of LhgR-based L-2-HG biosensor LHGFR in the absence or presence of L-2-HG. In particular, schematic representations of the tertiary structure of Venus and mTFP shown were predicted based on

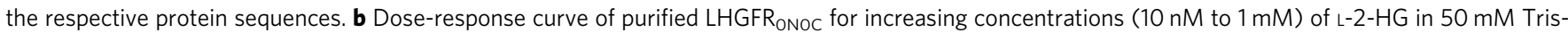
$\mathrm{HCl}$ buffer ( $\mathrm{pH}$ 7.4). The emission ratio of Venus to mTFP increased ( $430 \mathrm{~nm}$ excitation) after L-2-HG binding. $\mathbf{c}$ Heap map of the truncating the $\mathrm{N}$-terminal and C-terminal amino acids of LhgR to $\Delta R_{\max }$. The color indicates the value of $\Delta R_{\max }$ and white indicates the untested variants. $\mathbf{d}$ Comparison of the $\Delta R_{\text {max }}$ of a set of L-2-HG biosensor variants based on the C-terminal amino acid truncated of LhgR. e, $\mathbf{f}$ Specificities of the purified LHGFR $\mathrm{ON}_{\mathrm{OC}}$ (e) and LHGFR $\mathrm{ON7C}$ (f). The emission ratio changes of both biosensors were measured in the presence of $240 \mu \mathrm{M} D-$ lactate, L-lactate, L-2-HG, D-2-HG, or different intermediates of the TCA cycle and L-lysine catabolism. $\mathbf{g}$, $\mathbf{h}$ Reversal of L-2-HG binding with LHGFR by conversion of L-2-HG to 2-KG. The emission ratio of purified LHGFR $\mathrm{ON} 3 \mathrm{C}(\mathbf{g})$ and $\mathrm{LHGFR}_{\mathrm{ON} 7 \mathrm{C}}(\mathbf{h})$ was recorded in the absence and presence of $20 \mu \mathrm{M} \mathrm{L}-2-\mathrm{HG}$ before and after the addition of $5 \mu \mathrm{M}$ purified LhgO for $25 \mathrm{~min}$. All data shown are means \pm s.d. ( $n=3$ independent experiments).

LHGFR could detect possible variations of L-2-HG in living bacteria. $\mathrm{LHGFR}_{0 \mathrm{~N} 3 \mathrm{C}}$ and $\mathrm{LHGFR}_{3 \mathrm{~N} 7 \mathrm{C}}$, a control biosensor that did not respond to L-2-HG in vitro (Fig. 3c), were expressed in E. coli BL21 (DE3). Exogenous L-2-HG was added to the culture system of $E$. coli BL21(DE3) to achieve final concentrations between 0 and $10 \mathrm{mM}$ and the emission ratio was continuously recorded. As shown in Fig. 5a, exogenous L-2-HG could increase emission ratios of $\mathrm{LHGFR}_{0 \mathrm{~N} 3 \mathrm{C}}$ in a dose-dependent manner. The apparent $K_{d}$ of LHGFR $_{0 \mathrm{~N} 3 \mathrm{C}}$ expressed in E. coli BL21(DE3) was determined to be $891.72 \pm 32.10 \mu \mathrm{M}$ by fitting emission ratios against exogenous L-2-
HG concentrations (Fig. 5b). Maturation time lag and degradation of biosensors may be reasons responsible for higher apparent $K_{d}$ of LHGFR $_{0 \mathrm{~N} 3 \mathrm{C}}$ when expressed in $E$. coli ${ }^{35}$. The specificity of LHGFR $_{0 \mathrm{~N} 3 \mathrm{C}}$ expressed in E. coli BL21(DE3) was also characterized. As shown in Fig. 5c, only exogenous L-2-HG could significantly increase the emission ratio in E. coli BL21(DE3), while glutarate, D2-HG, and glucose could not.

Besides being a metabolic intermediate of exogenous glutarate catabolism in P. putida KT2440, L-2-HG is also reported as a metabolite produced from endogenous L-lysine during carbon 

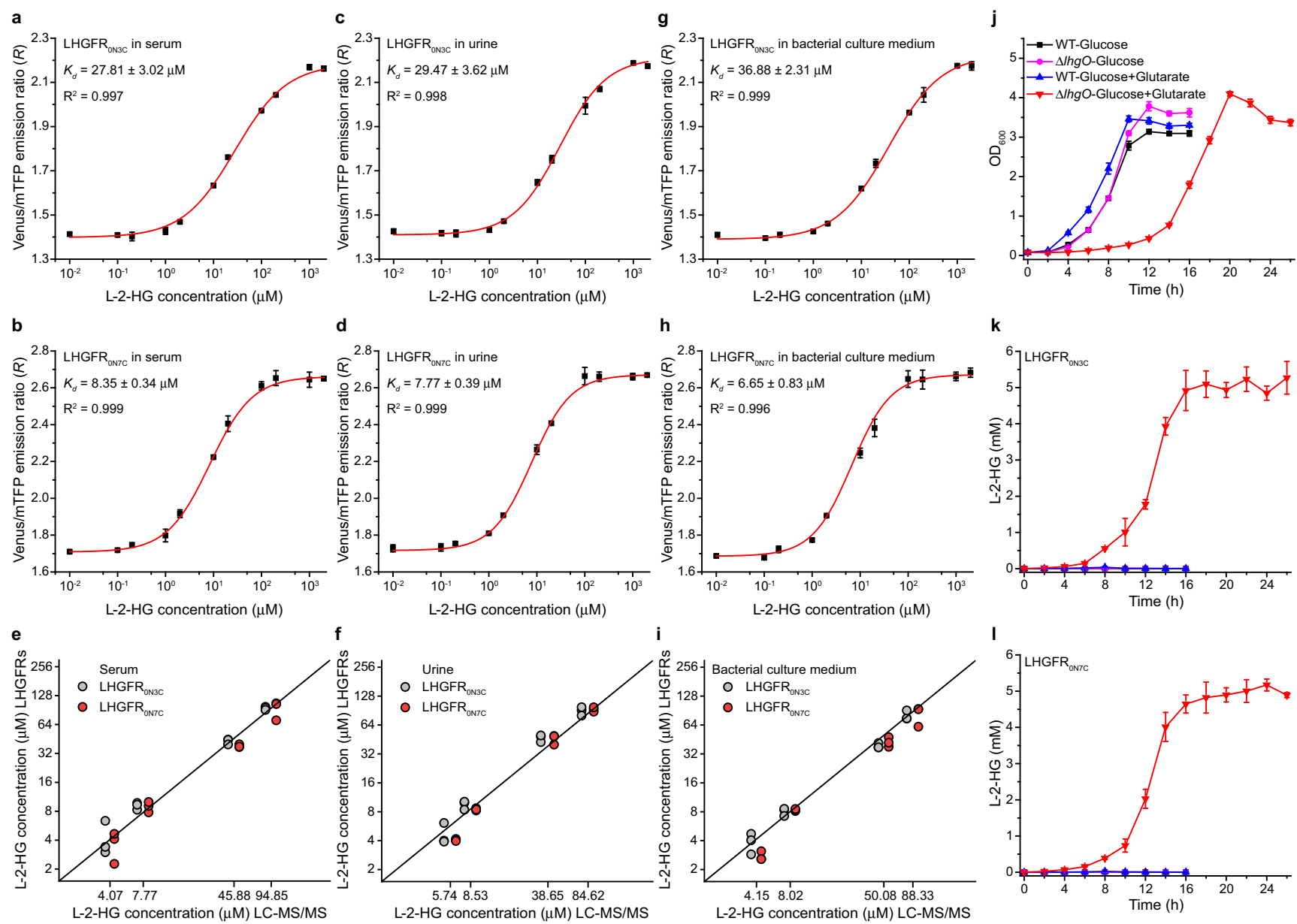

Fig. 4 Validation of purified LHGFR for determination L-2-HG levels in body fluids and bacterial culture system. a-d Dose-response curves of purified LHGFR $_{O N 3 C}$ and LHGFR ${ }_{O N 7 C}$ for increasing concentrations (10 $\mathrm{nM}$ to $2 \mathrm{mM}$ ) of L-2-HG in serum $(\mathbf{a}, \mathbf{b})$ and urine $(\mathbf{c}$, d). e, $\mathbf{f}$ Comparison between the quantitative results of L-2-HG added in serum (e) and urine (f) by LC-MS/MS and LHGFR. The gray circles and red circles represent the quantitative

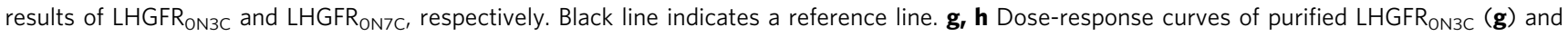
LHGFR $_{\text {ON7C }}(\mathbf{h})$ for increasing concentrations ( $10 \mathrm{nM}$ to $2 \mathrm{mM}$ ) of L-2-HG in bacterial culture medium. i Comparison between the quantitative results of L2-HG in bacterial culture medium by LC-MS/MS and LHGFR. $\mathbf{j}$ Growth of $P$. putida KT2440 and its IhgO mutant in medium containing 20 mM glucose and $5 \mathrm{mM}$ glutarate as the carbon sources. k, I Determination of extracellular L-2-HG accumulation of $P$. putida KT2440 and its IhgO mutant by purified LHGFR ON3C $_{\text {Ok }}$ and LHGFR 0 ONC $(\mathbf{I})$. All data shown are means \pm s.d. $(n=3$ independent experiments).

starvation of $E$. coli ${ }^{36,37}$. Thus, whether carbon starvation could induce intracellular $\mathrm{L}-2-\mathrm{HG}$ accumulation of $E$. coli was investigated. As shown in Fig. 5d, no change in the emission ratio was detected when $20 \mathrm{mM}$ glucose was added to the culture system. However, the emission ratio increased during carbon starvation of E. coli BL21(DE3), suggesting that carbon starvation induced L-2-HG production. The emission ratios also increased after culturing $E$. coli cells for $6 \mathrm{~h}$ with glucose addition (Fig. 5e), which might be due to carbon starvation induced by depletion of exogenous glucose. In addition, the emission ratio increased at the beginning of carbon starvation, reaching a maximum value at $3 \mathrm{~h}$ and then decreased to initial levels at $8 \mathrm{~h}$ (Fig. 5e). These results confirmed that $\mathrm{L}-2-\mathrm{HG}$ is a temporary metabolite during carbon starvation and $\mathrm{LHGFR}_{0 \mathrm{~N} 3 \mathrm{C}}$ can be used to monitor the real-time change in intracellular L-2-HG concentrations.

To identify whether carbon starvation-induced endogenous L-2-HG production also results from the glutarate hydroxylase activity of CsiD, gene $c s i D$ was disrupted and $\mathrm{LHGFR}_{0 \mathrm{~N} 3 \mathrm{C}}$ was expressed in E. coli MG1655(DE3). Gene lhgO in E. coli MG1655 (DE3) was also disrupted to investigate its role in endogenous L-2-HG catabolism. As expected, the emission ratio of
LHGFR $_{0 \mathrm{~N} 3 \mathrm{C}}$ in $E$. coli MG1655(DE3) $(\Delta c s i D)$ remained unaffected during carbon starvation, whereas disruption of $\operatorname{lhgO}$ significantly increased the emission ratio of $\mathrm{LHGFR}_{0 \mathrm{~N} 3 \mathrm{C}}$ in $E$. coli MG1655(DE3) ( $\Delta l h g O)$ (Fig. 5f), indicating the roles of CsiD and LhgO in endogenous L-2-HG metabolism during carbon starvation. The performance of LHGFR $_{0 N 7 C}$ in monitoring $\mathrm{L}-2-\mathrm{HG}$ fluctuations in living bacteria was also studied and similar results were acquired (Supplementary Fig. 13). As for the control biosensor LHGFR $_{3 \mathrm{~N} 7 \mathrm{C}}$, no change of emission ratio could be detected in living bacteria for any of the above-mentioned experiments (Supplementary Fig. 14).

Monitoring L-2-HG production in human cells by LHGFR. Next, LHGFR ${ }_{0 \mathrm{~N} 3 \mathrm{C}}, \mathrm{LHGFR}_{0 \mathrm{N7C}}$, and LHGFR $_{3 \mathrm{~N} 7 \mathrm{C}}$ were expressed in the cytosol of HEK293FT cells. The addition of $10 \mathrm{mM} \mathrm{L-2-HG}$ affected mTFP and Venus fluorescence intensities, which caused a non-uniform increase in the emission ratio of $\mathrm{LHGFR}_{0 \mathrm{~N} 3 \mathrm{C}^{-}}$ expressing single cell (Fig. 6a and Supplementary Movie). The average emission ratio reached a maximum value at $5 \mathrm{~min}$ and remained constant during the subsequent confocal imaging period (Fig. 6b). The apparent $K_{d}$ of $\mathrm{LHGFR}_{0 \mathrm{~N} 3 \mathrm{C}}$ expressed in 


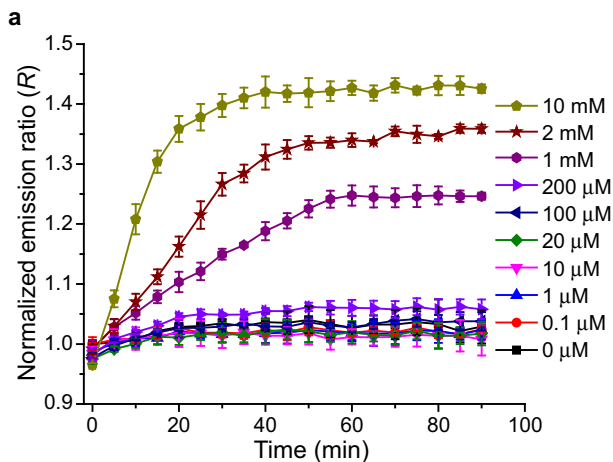

d

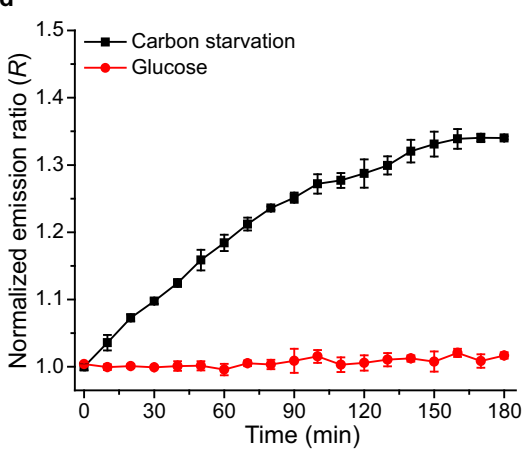

b
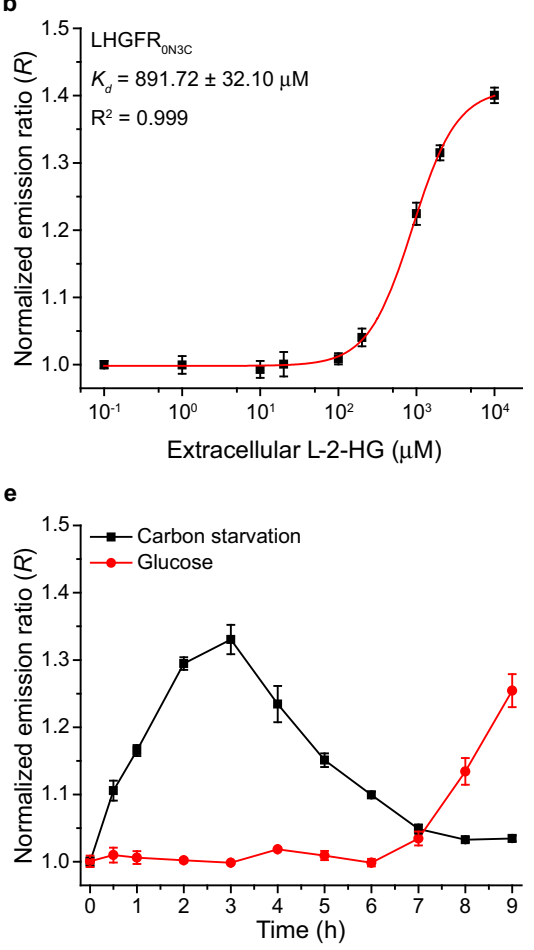
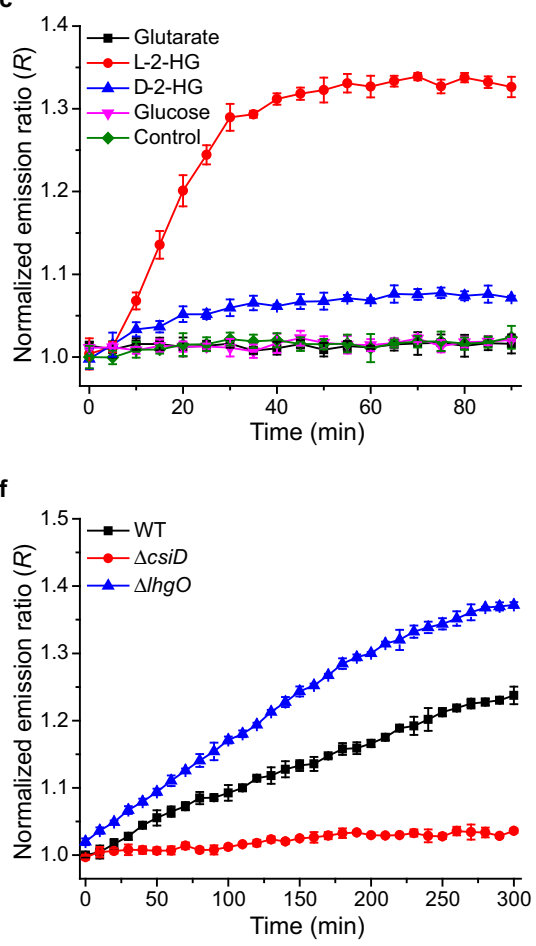

Fig. 5 Monitoring L-2-HG fluctuations in living bacteria by LHGFRon3c. a Time course of the emission ratio changes of LHGFRoN3C expressed in E. coli BL21(DE3) in response to exogenous L-2-HG addition. All ratios were normalized to the control (ratio in the absence of L-2-HG at time point zero). b Normalized dose-response curve of LHGFR ${ }_{0 N 3 C}$ expressed in E. coli BL21(DE3) for increasing concentrations (100 nM to $10 \mathrm{mM}$ ) of L-2-HG at time point 60 min. c Time course of the emission ratio changes of LHGFRoN3c expressed in E. coli BL21(DE3) in response to the addition of $1 \mathrm{mM}$ glutarate, L-2-HG, $\mathrm{D}-2-\mathrm{HG}$, or glucose. All data were normalized to the control (ratio in the absence of any tested compounds at time point zero). $\mathbf{d}$ Detection of carbon starvation-induced L-2-HG accumulation over time by LHGFR ON3C $_{3}$ expressed in E. coli BL21(DE3). Emission ratio changes of LHGFR ON3C were measured when cultured in carbon starvation medium (black line) and medium with $20 \mathrm{mM}$ glucose (red line). All data were normalized to samples under carbon

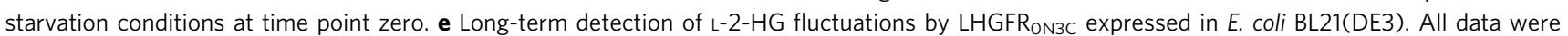
normalized to samples under carbon starvation conditions at time point zero. $\mathbf{f}$ Identification of the roles of CsiD and LhgO in endogenous L-2-HG

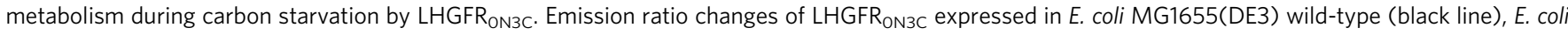
MG1655(DE3) ( $\Delta$ csiD) (red line), and E. coli MG1655(DE3) ( $\Delta / \mathrm{hgO}$ ) (blue line) were measured in carbon starvation medium. All data were normalized to time point zero of the wild-type strain. Inconsistent initial emission ratios were detected in bacterial cells under different conditions. The normalized emission ratios were thus used to monitor the changes of L-2-HG in different bacterial cells. All data shown are means \pm s.d. $(n=3$ independent experiments).

HEK293FT cells for L-2-HG was determined to be $43.79 \pm 3.05$ $\mu \mathrm{M}$ (Fig. 6c). Based on the emission ratio of non-permeabilized HEK293FT cells under physiological conditions, the basal L-2-HG concentration in $\mathrm{LHGFR}_{0 \mathrm{~N} 3 \mathrm{C}^{-}}$-expressing cells was $22.95 \pm 11.22$ $\mu \mathrm{M}$ (Fig. 6c). Only exogenous L-2-HG could significantly increase the emission ratio of $\mathrm{LHGFR}_{0 \mathrm{~N} 3 \mathrm{C}}$ in $10 \mu \mathrm{M}$ digitoninpermeabilized HEK293FT cells, suggesting the specificity of the biosensor inside living human cells (Fig. 6d). L2HGDH, the only reported enzyme that is able to catabolize L-2-HG in human cells, is localized in mitochondria ${ }^{13}$. The mitochondrial targeting sequence was appended to $\mathrm{LHGFR}_{\mathrm{ON} 3 \mathrm{C}}$ to localize the biosensor in mitochondria (Supplementary Fig. 15a). Exogenous L-2-HG also induced an increase in the emission ratio of mitochondrial LHGFR $_{0 \mathrm{~N} 3 \mathrm{C}}$ (Supplementary Fig. 15b). The emission ratio of LHGFR $_{0 \mathrm{~N} 3 \mathrm{C}}$ localized in mitochondria was similar to that of in cytosol (Supplementary Fig. 15c). The uniform distribution of L-2-HG confirmed the presence of a transporter responding for the transport of L-2-HG between cytosol and mitochondria.

HEK293FT cells were then co-transfected with siRNA targeting L2HGDH and $\mathrm{LHGFR}_{0 \mathrm{~N} 3 \mathrm{C}}$. As shown in Fig. 6e, the transfection of siRNA targeting $\mathrm{L} 2 \mathrm{HGDH}$ increased the emission ratio of $\mathrm{LHGFR}_{0 \mathrm{~N} 3 \mathrm{C}}$, indicating an accumulation of intracellular $\mathrm{L}-2-\mathrm{HG}$ due to a decrease in L2HGDH levels. Cells with different emission ratios could be easily distinguished in a mixture of HEK293FT cells with or without L2HGDH knockdown (Supplementary Fig. 16a-b). As expected, overexpression of L2HGDH decreased the emission ratio of $\mathrm{LHGFR}_{0 \mathrm{~N} 3 \mathrm{C}}$, further supporting the function of $\mathrm{L} 2 \mathrm{HGDH}$ in L-2-HG catabolism (Supplementary Fig. 16c).

The response of LHGFR $_{0 \mathrm{~N} 3 \mathrm{C}}$ to changes in hypoxia-induced production of L-2-HG in human cells was also studied. The emission ratio of $\mathrm{LHGFR}_{0 \mathrm{~N} 3 \mathrm{C}}$ in HEK293FT cells after $24 \mathrm{~h}$ exposure to $2 \%$ oxygen was higher than the ratio obtained under normoxic conditions and hypoxia induced a 3.5 -fold increase in the concentration of L-2-HG (Fig. 6f). In addition, exogenous cellpermeable dimethyl-2-ketoglutarate significantly increased the emission ratio of $\mathrm{LHGFR}_{0 \mathrm{~N} 3 \mathrm{C}}$ under hypoxic conditions, suggesting that hypoxia-induced L-2-HG might originate from 2-KG (Fig. 6f).

LDHA and $\mathrm{MDH} 2$ have been reported to participate in hypoxia-induced L-2-HG production due to their "promiscuous" catalytic activities ${ }^{1-4}$. In support of this conclusion, siRNAs targeting LDHA and MDH2 were transfected separately or in combination into LHGFR $_{0 \mathrm{~N} 3 \mathrm{C}}$-expressing HEK293FT cells. As shown in Fig. 6g, the decrease of LDHA and $\mathrm{MDH} 2$ reduced the emission ratio of $\mathrm{LHGFR}_{0 \mathrm{~N} 3 \mathrm{C}}$ under hypoxic conditions, suggesting that these enzymes indeed contribute to the production of $\mathrm{L}-2-\mathrm{HG}$ from $2-\mathrm{KG}$. 

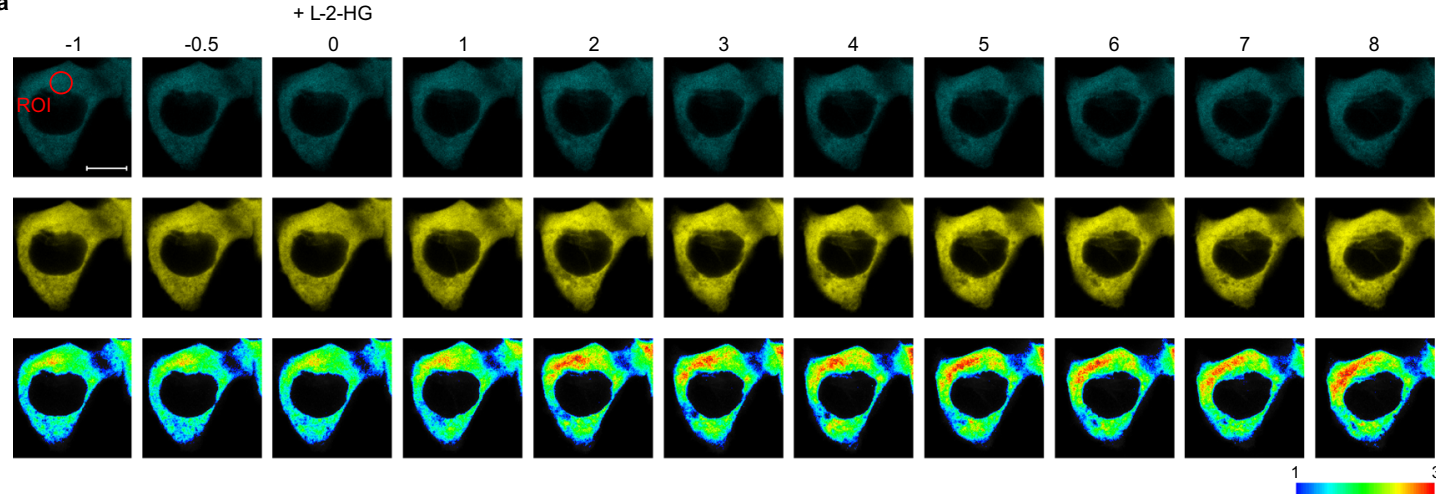

b

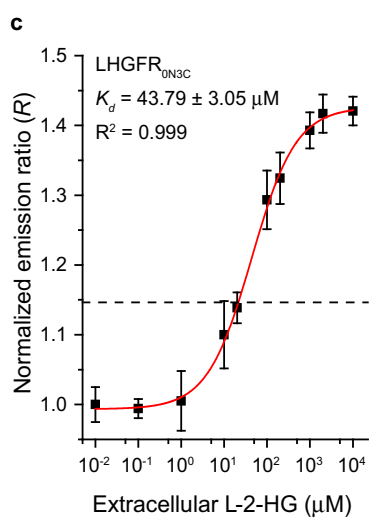

c
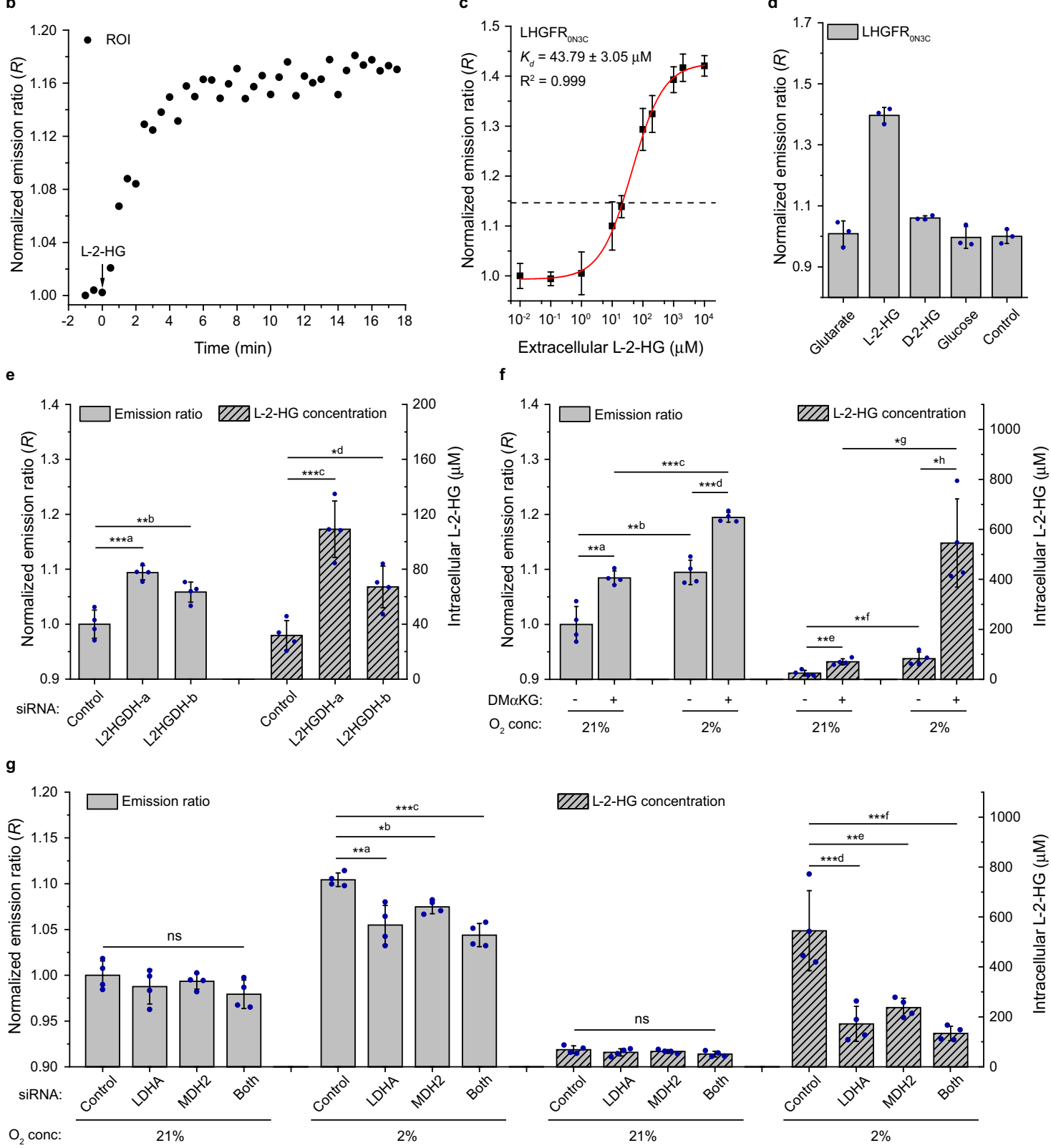

The performance of LHGFR $_{0 N 7 C}$ in monitoring $\mathrm{L}-2-\mathrm{HG}$ fluctuations in living HEK293FT cells was also studied and similar results were acquired (Supplementary Fig. 17). In addition, the emission ratio of control cells expressing LHGFR $_{3 \mathrm{~N} 7 \mathrm{C}}$ remained unaffected by exogenous L-2-HG addition and hypoxia treatment (Supplementary Fig. 18).

\section{Discussion}

Bacteria have evolved to respond and catabolize a wide range of metabolites. The availability of genomic information from different organisms provided researchers with a new route to predict transcriptional regulators and their physiological functions. In this study, we used a genomic neighborhood analysis approach 


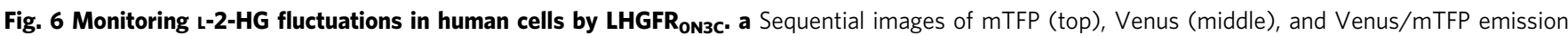
ratio (bottom, pseudocolored) of single HEK293FT cell expressing LHGFR ON3C. $10 \mathrm{mM} \mathrm{L-2-HG} \mathrm{was} \mathrm{added} \mathrm{at} \mathrm{time} \mathrm{point} \mathrm{zero} \mathrm{(min).} \mathrm{Elapsed} \mathrm{time} \mathrm{(in}$ minutes) after the addition of L-2-HG is shown at the top of the images. Scale bar, $10 \mu \mathrm{m}$. $\mathbf{b}$ Time course of the emission ratio changes inside the region of

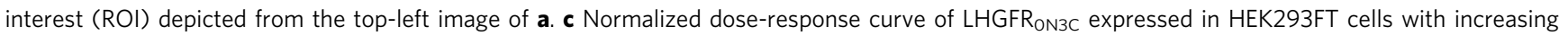
concentrations ( $10 \mathrm{nM}$ to $10 \mathrm{mM}$ ) of L-2-HG. Cells were permeabilized with $10 \mu \mathrm{M}$ digitonin. The emission ratio of non-permeabilized HEK293FT cells

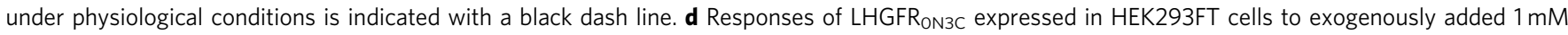
glutarate, L-2-HG, D-2-HG, and glucose. Cells were permeabilized with $10 \mu \mathrm{M}$ digitonin. All data were normalized to the control (ratio in the absence of any tested compounds). e Identification of the function of L2HGDH in L-2-HG catabolism by LHGFRoN3c. The emission ratio was measured after co-transfecting siRNA targeting L2HGDH and LHGFR ON3C for $48 \mathrm{~h}$. ${ }^{\star \star \star} \mathrm{a}: P=0.0002,{ }^{\star \star} \mathrm{b}: P=0.0058,{ }^{\star \star \star}{ }^{\circ} \mathrm{C}: P=0.0002,{ }^{\star} \mathrm{d}: P=0.0303$. $\mathrm{f}$ Detection of hypoxia-induced $\mathrm{L}-$ 2-HG accumulation by LHGFR ${ }_{0 N 3 C}$. Emission ratio changes were recorded after LHGFR ${ }_{0 N 3 C}$-expressing HEK293FT cells cultured in normoxia or hypoxia in the absence and presence of $5 \mathrm{mM}$ dimethyl-2-ketoglutarate (DM $\mathrm{DKG}$ ) for $24 \mathrm{~h}$. The emission ratio was normalized to normoxic conditions without DMoKG. ${ }^{\star \star}$ a: $P=0.0029,{ }^{\star \star}$ b: $P=0.0030,{ }^{\star \star \star}{ }^{\star} \mathrm{c}: P<0.0001,{ }^{\star \star \star} \mathrm{d}: P=0.0002,{ }^{\star \star} \mathrm{e}: P=0.0016,{ }^{\star \star} \mathrm{f}: P=0.0068,{ }^{\star} \mathrm{g}: P=0.0126,{ }^{\star} \mathrm{h}: P=0.0139 . \mathbf{g}$ Identification of the functions of LDHA and MDH2 in L-2-HG anabolism by LHGFR ${ }_{0 N 3 C}$. HEK293FT cells were cultured in the presence of $5 \mathrm{mM}$ DM $\alpha$ KG. The emission ratio was normalized to the normoxic conditions treated with negative siRNA. ${ }^{\star \star} \mathrm{a}: P=0.0011,{ }^{\star} \mathrm{b}: P=0.0400,{ }^{\star \star \star} \mathrm{c}: P=0.0002,{ }^{\star \star \star} \mathrm{d}: P=$ $0.0004,{ }^{\star \star} \mathrm{e}: P=0.0021,{ }^{\star \star \star} \mathrm{f}: P=0.0002$. Inconsistent initial emission ratios were detected in HEK293FT cells under different conditions. The normalized emission ratios were thus used to monitor the changes of $\mathrm{L}-2-\mathrm{HG}$ in different HEK293FT cells. All data shown are means $\pm \mathrm{s}$.d. $(n=3,3,4,4$, and 4 independent experiments for $\mathbf{c}, \mathbf{d}, \mathbf{e}, \mathbf{f}$, and $\mathbf{g})$. ${ }^{\star} P<0.05$; ${ }^{\star \star} P<0.01$; ${ }^{\star \star \star} P<0.001$; ns, no significant difference $(P \geq 0.05)$; one-way ANOVA test with Tukey's Multiple Comparison Test for $\mathbf{e}$ and $\mathbf{g}$; two-tailed $t$-test for $\mathbf{f}$.

combined with genetic and biochemical techniques to discover the transcriptional regulator of L-2-HG catabolism. The identified transcriptional regulator, LhgR, was present directly upstream of LhgO in P. putida W619 (Fig. 1a). It specifically binds to the promoter region of $l h g O$ and represses the transcription of $l h g O$ gene. L-2-HG interferes with the DNA-binding activity of LhgR and induces expression of LhgO. LhgR is an example of the bacterial allosteric transcription factor that specially responds to L-2-HG. This finding showed the application of a collection of sequenced genomes in the identification of transcriptional regulators and the approach can be expanded to target other transcriptional regulators in diverse bacteria.

$\mathrm{L}-2-\mathrm{HG}$ is a harbinger of altered metabolism and participates in the pathogenesis of L-2-hydroxyglutaric acidurias and cancer ${ }^{12-15,38,39}$. Standard methods to measure L-2-HG are based on MS techniques that are time-consuming and require highly skilled workers. LhgR can bind to $\mathrm{L}-2-\mathrm{HG}$ and then undergo conformational changes, which in turn affects DNA-binding. Thus, a FRET biosensor, LHGFR $_{0 \text { NoC, }}$ utilizing the allosteric transcription factor LhgR as an L-2-HG biorecognition element was constructed for a convenient assay of $\mathrm{L}-2-\mathrm{HG}$ concentrations. As a ratiometric sensor, the emission ratio changes of LHGFR are not affected by the amount of sensor in biological samples or in living cells, and thus allows more accurate measurements. The biosensor was optimized by truncating the $\mathrm{N}$-terminal and $\mathrm{C}$ terminal domains of LhgR or by adding an artificial linker to the $\mathrm{N}$-terminal and C-terminal regions of LhgR. The optimized variants, $\mathrm{LHGFR}_{0 \mathrm{~N} 3 \mathrm{C}}$ and $\mathrm{LHGFR}_{0 \mathrm{~N} 7 \mathrm{C}}$, increased $\Delta R_{\max }$ from $11.47 \pm 0.38 \%$ to $56.13 \pm 0.29 \%$ and $60.37 \pm 1.30 \%$, respectively, with significantly improved sensitivity for L-2-HG detection (Fig. 3b, Supplementary Fig. 6a and Fig. 6e). Besides signal recognition, signal transduction is also an essential aspect of the development of biosensors. Various biosensing systems, including CRISPR-Cas12a- and allosteric transcription factors-mediated small molecule detector (CaT-SMelor) ${ }^{40}$, allosteric transcription factors-based nicked DNA-template-assisted signal transduction (aTF-NAST) ${ }^{41}$, and quantum-dot-allosteric transcription factorsFRET $^{42}$, have been reported. Other biosensors based on L-2-HG responding LhgR and the corresponding transduction mechanisms could also be developed for the detection of L-2-HG.

$\mathrm{L}-2-\mathrm{HG}$ is a biomarker for L-2-hydroxyglutaric aciduria and a variety of cancers. The rapid, sensitive, and specific measurement of L-2-HG in body fluids is of clinical significance ${ }^{12-15,38,43}$. The LODs of LHGFR $\mathrm{LN} 3 \mathrm{C}_{\mathrm{C}}$ and $\mathrm{LHGFR}_{0 \mathrm{~N} 7 \mathrm{C}}$ for $\mathrm{L}-2-\mathrm{HG}$ in serum and urine were $5.84 \mu \mathrm{M}$ and $15.74 \mu \mathrm{M}, 1.68 \mu \mathrm{M}$ and $0.92 \mu \mathrm{M}$, respectively (Supplementary Table 3). The reported concentration of $\mathrm{L}-2-\mathrm{HG}$ in plasma of patients with L-2-hydroxyglutaric aciduria and L-2-HG-associated brain malignancies is about 7-84 $\mu \mathrm{M}^{38,43}$. Thus, these biosensors especially $\mathrm{LHGFR}_{0 \mathrm{N7C}}$ are suitable for the measurement of the endogenous L-2-HG in body fluids of patients with L-2-HG related diseases. D-2-HG concentration in the urine, plasma, and cerebrospinal fluid of patients with combined D,L-2-hydroxyglutaric aciduria or D2HGDH mutation-associated D-2-hydroxyglutaric aciduria was reported to be less than $100 \mu \mathrm{M}^{38}$. Serum 2-HG in IDH1-mutated and IDH2-mutated cancers like acute myeloid leukemia is about $300 \mu \mathrm{M}^{44}$. LHGFR ${ }_{0 \mathrm{~N} 3 \mathrm{C}}$ has high LODs for D-2-HG in serum and urine $(781.90 \mu \mathrm{M}$ and $3876.40 \mu \mathrm{M})$, which can prevent the falsepositive results in patients with $\mathrm{D}$-2-hydroxyglutaric aciduria and/ or IDH-mutant cancers (Supplementary Table 4). Compared with LHGFR $_{0 \mathrm{~N} 3 \mathrm{C}}$, LHGFR 0 N7C has a higher affinity with D-2-HG. However, this biosensor is a sensitive variant with low LOD for L-2-HG (Supplementary Table 3). Assessment of L-2-HG by LHGFR $_{0 \mathrm{~N} 7 \mathrm{C}}$ can be easily conducted after a simple dilution to prevent the possible interference induced by $\mathrm{D}-2$-HG-related diseases. Besides higher sensitivity and specificity, LHGFR $_{0 \mathrm{~N} 3 \mathrm{C}}$ and LHGFR $_{0 \mathrm{~N} 7 \mathrm{C}}$ also have superior accuracy and precision over previous MS-based methods for L-2-HG detection (Fig. 4 and Supplementary Table 2). Being genetically encoded, LHGFR $_{0 \mathrm{~N} 3 \mathrm{C}}$ and LHGFR $_{0 \mathrm{~N} 7 \mathrm{C}}$ could be produced in great quantities by recombinant bacteria with low cost and could be applied in future rapid and sensitive clinical diagnosis of L-2-HG-related diseases.

L-2-HG plays important roles in diverse physiological processes such as hypoxic adaption, immunity, and tumorigenesis, and the establishment of the intracellular detection method of this metabolite is of great research significance ${ }^{3,12,14,16}$. Intracellular L-2-HG concentrations of activated $\mathrm{CD}^{+}{ }^{+} \mathrm{T}$-lymphocytes, renal cancer cells, and various cells under hypoxic conditions were about $25 \mu \mathrm{M}$ to several hundred micromoles $3,12,16$. Compared with LHGFR $_{0 \mathrm{~N} 7 \mathrm{C}}$, $\mathrm{LHGFR}_{0 \mathrm{~N} 3 \mathrm{C}}$ with a higher $K_{d}$ for $\mathrm{L}-2-\mathrm{HG}$ $(29.33 \pm 1.24 \mu \mathrm{M})$ is a more viable alternative for the detection of intracellular L-2-HG (Supplementary Fig. 6a and Fig. 6e, Supplementary Table 1). Based on the in vivo response curves of LHGFR $_{0 \mathrm{~N} 3 \mathrm{C}}$, the basal L-2-HG concentration in HEK293FT cells under physiological conditions was determined to be $22.95 \pm$ $11.22 \mu \mathrm{M}$ (Fig. 6c), which is similar to results acquired using MSbased approaches after complicated sample handling and data analysis $^{2,3}$. LHGFR ${ }_{0 \mathrm{~N} 3 \mathrm{C}}$ also has a low affinity with D-2-HG. The LOD of LHGFR ${ }_{0 N 3 C}$ for D-2-HG was $872.59 \mu \mathrm{M}$ and the $K_{d}$ value could not be determined accurately because of the relatively low 
affinity (Supplementary Fig. 9c and Supplementary Table 1). D-2$\mathrm{HG}$ at a concentration of $240 \mu \mathrm{M}$, which is much higher than its basal intracellular concentrations, could barely affect the response of LHGFR ON3C $_{\text {L }}$ to-2-HG (Supplementary Fig. 9e). IDH1-R132H mutation can lead to an extreme intracellular D-2-HG accumulation at millimolar levels ${ }^{45}$. The expression of IDH1-R132H resulted in a $4.9 \%$ increase in the emission ratio of $\mathrm{LHGFR}_{0 \mathrm{~N} 3 \mathrm{C}}$ under extreme conditions (Supplementary Fig. 19). Calibrated dose-response curves of $\mathrm{LHGFR}_{0 \mathrm{~N} 3 \mathrm{C}}$ for $\mathrm{L}-2-\mathrm{HG}$ would be required for monitoring the fluctuations of $\mathrm{L}-2-\mathrm{HG}$ in cells with IDH mutations.

The potential of LHGFR for real-time monitoring of fluctuations in intracellular L-2-HG concentrations was illustrated by using bacterial cells and HEK293FT cells. It was revealed that carbon starvation also induced temporary intracellular accumulation of L-2-HG in E. coli cells. CsiD and LhgO played indispensable roles in endogenous anabolism and catabolism of L-2HG, respectively (Fig. $5 \mathrm{f}$ and Supplementary Fig. 13f). In addition, it was identified that the growth of the strain containing lhgO mutation was inhibited when high levels of L-2-HG were present (Fig. 4j-1). Besides being a pathogenic metabolite inducing various cancers and L-2-hydroxyglutaric aciduria in humans $^{12-15,38,43}$, L-2-HG would also be a toxic metabolite to bacterial cells. L-2-HG catabolizing enzymes, including L2HGDH in humans ${ }^{9}$, dL2HGDH in Drosophila ${ }^{17}$, and LhgO in P. putida ${ }^{7,8}$ and $E$. coli ${ }^{6,46}$, might all exist as detoxification proteins of $\mathrm{L}-2-\mathrm{HG}$. The functions of L2HGDH in L-2-HG catabolism and LDHA and $\mathrm{MDH} 2-$ mediated 2-KG reduction in hypoxia-induced $\mathrm{L}-2-\mathrm{HG}$ production were also confirmed in HEK293FT cells using LHGFR as an indicator of L-2-HG. LHGFR can be added to the emerging list of metabolite sensors that have been established in mammalian cells, such as probes for $\mathrm{ATP}^{47}$, acetylcholine ${ }^{48}$, glycine $\mathrm{e}^{24}$, and $\mathrm{NAD}^{+} / \mathrm{NADH}^{49}$. Several genetically encoded fluorescent metabolite sensors, like the $\mathrm{NAD}^{+} / \mathrm{NADH}$ probe SoNar, have been successfully applied in the screening of anti-tumor agents ${ }^{49}$. L-2-HG has been exploited as a potential therapeutic target in renal cancer ${ }^{14}$ or a biomarker for cancer diagnosis and prognostic assessment ${ }^{15,50}$. The $\mathrm{L}-2-\mathrm{HG}$ biosensors might also be utilized in the diagnosis and screening of anti-tumor agents for L-2-HGrelated cancer.

In summary, a regulatory protein LhgR, which is involved in L-2-HG catabolism and specifically responds to L-2-HG, was identified in P. putida W619. Two LhgR-based L-2-HG biosensors, $\mathrm{LHGFR}_{0 \mathrm{~N} 3 \mathrm{C}}$ and $\mathrm{LHGFR}_{0 \mathrm{~N} 7 \mathrm{C}}$, with high sensitivity, specificity, and stability, were then constructed. The methods for quantitative estimation of $\mathrm{L}-2-\mathrm{HG}$ concentrations in various biological samples and living cells by using L-2-HG biosensors were also established. We expect these LhgR-based L-2-HG biosensors to be of practical interest in future research on the metabolism of L-2-HG and the diagnosis and treatment of L-2-HG-related diseases.

\section{Methods}

Bacterial strains and culture conditions. The bacterial strains used in this study are listed in Supplementary Data 1. E. coli and its derivatives were cultured in Luria-Bertani (LB) broth at $37^{\circ} \mathrm{C}$ and $180 \mathrm{rpm}$. P. putida KT2440 and its derivatives were grown in minimal salt mediums (MSMs) containing different carbon sources at $30^{\circ} \mathrm{C}$ and $200 \mathrm{rpm}$. Antibiotics were used at the following concentrations: tetracycline at $30 \mu \mathrm{g} \mathrm{mL}^{-1}$; kanamycin at $50 \mu \mathrm{g} \mathrm{mL}^{-1}$; ampicillin at $100 \mu \mathrm{g} \mathrm{mL}^{-1}$; spectinomycin, at $50 \mu \mathrm{g} \mathrm{mL}^{-1}$; and chloramphenicol at $40 \mu \mathrm{g} \mathrm{mL}^{-1}$.

Cloning of F2-IhgR-F1-IhgO and IhgO. All the plasmids and primers used in this study are listed in Supplementary Data 1 and Data 2, respectively. The gene segment F2-lhgR-F1-lhgO of $P$. putida W619 was synthesized by Tongyong Biosystem Co., Ltd (China). The lhgO gene of P. putida W619 was amplified and cloned into pME6032 plasmid using the restriction sites of EcoRI and KpnI to construct pME6032-lhgO, and the $P_{t a c}$ promoter of pME6032 was replaced by the gene segment F2-lhgR-F1-lhgO using the restriction sites of SacI and BamHI to construct pME6032-F2-lhgR-F1-lhgO, then both recombinant plasmids were transferred into different derivatives of $P$. putida KT2440 by electroporation, respectively.

Construction of $P$. putida KT2440 and E. coli MG1655(DE3) mutants. Genes of $P$. putida KT2440 were deleted via allele exchange using the pK18 mobsacB system $^{51}$. Briefly, the homologous arms upstream and downstream of the target gene were PCR amplified and fused together by recombinant PCR. The generated fusion fragment was cloned into the suicide plasmid pK18mobsacB. The resulting plasmid was transferred into $P$. putida KT2440 by electroporation. The single crossover cells and the second crossover cells were sequentially screened from LB plates containing $50 \mu \mathrm{g} \mathrm{mL}^{-1}$ kanamycin or $10 \%$ (wt/vol) sucrose, respectively.

To construct the E. coli MG1655(DE3) ( $\Delta c s i D)$ mutant strain, the homologous arm upstream of the $c s i D$ gene, kanamycin resistance cassette, and the homologous arm downstream of the csiD gene were PCR amplified using the primers $c s i D-F 1 /$ $c s i D-\mathrm{R} 1, c s i D-\mathrm{F} 2 / c s i D-\mathrm{R} 2$, and $c s i D-\mathrm{F} 3 / c s i D-\mathrm{R} 3$, respectively. The PCR products were fused together by recombinant PCR, and the resulting fusion was transferred into E. coli MG1655(DE3) harboring pTKRed plasmid following isopropyl- $\beta$-D-1thiogalactopyranoside (IPTG) induction. The recombinant cells were selected on LB plates containing $50 \mu \mathrm{g} \mathrm{mL}{ }^{-1}$ kanamycin at $37^{\circ} \mathrm{C}$. The pCP20 plasmid was transferred into the selected cells, followed by a second screening on LB plates containing $40 \mu \mathrm{g} \mathrm{mL}^{-1}$ chloramphenicol at $30^{\circ} \mathrm{C}$, then cultured in $\mathrm{LB}$ medium at $42{ }^{\circ} \mathrm{C}$ to eliminate pCP20 plasmid. The $l h g O$ mutant of $E$. coli MG1655(DE3) was generated by the same process. All mutants were verified by PCR and sequencing.

Enzymatic assay of LhgO. The derivatives of $P$. putida KT2440 were cultured in $50 \mathrm{~mL}$ MSMs with $5 \mathrm{~g} \mathrm{~L}^{-1}$ different compounds as carbon sources at $30^{\circ} \mathrm{C}$ and $200 \mathrm{rpm}$. The cells were harvested at mid-log phase, washed twice and resuspended in phosphate-buffered saline (PBS), then lysed by sonication on ice after the addition of $1 \mathrm{mM}$ phenylmethylsulfonyl fluoride (PMSF). The supernatants obtained were used for further enzyme activity measurements after a centrifugation process $\left(13,000 \times g\right.$ for $10 \mathrm{~min}$ at $\left.4^{\circ} \mathrm{C}\right)$. Protein concentrations of the supernatants were determined using the Bradford protein assay kit (Sangon, China).

The activity of LhgO was assayed at $30^{\circ} \mathrm{C}$ by monitoring the reduction of dichlorophenol-indophenol (DCPIP) corresponding to the change of absorbance at $600 \mathrm{~nm}$ using a UV/visible spectrophotometer (Ultrospec 2100 pro, Amersham Biosciences, USA). The $800 \mu \mathrm{L}$ reaction solution contained $0.1 \mathrm{mM} \mathrm{L-2-HG}$, $0.05 \mathrm{mM}$ DCPIP, $0.2 \mathrm{mM}$ phenazine methosulfate (PMS) in PBS and $40 \mu \mathrm{L}$ crude extracts. One unit of LhgO activity was defined as the amount of enzyme that catalyzed the reduction of $1 \mu \mathrm{mol}$ of DCPIP per minute.

Expression, purification, and characterization of LhgR. To express and purify the recombinant LhgR, the $l h g R$ gene was PCR amplified using the primer pair lhgR-F/lhgR-R, which contained BamHI and HindIII restriction sites, respectively, and then cloned into the pETDuet-1 plasmid to construct pETDuet-lhgR. The $E$. coli $\mathrm{BL} 21$ (DE3) strains harboring pETDuet-lhgR plasmid were grown to an $\mathrm{OD}_{600}$ of 0.6 in $\mathrm{LB}$ medium at $37^{\circ} \mathrm{C}$, after which the cells were induced for $12 \mathrm{~h}$ with $1 \mathrm{mM}$ IPTG at $16^{\circ} \mathrm{C}$. The cells were harvested, washed twice, and resuspended in

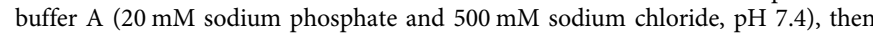
lysed by sonication on ice after the addition of $1 \mathrm{mM}$ PMSF and $10 \%$ ( $\mathrm{vol} / \mathrm{vol}$ ) glycerol. The cell lysate was centrifuged at $13,000 \times g$ for $40 \mathrm{~min}$ at $4{ }^{\circ} \mathrm{C}$, and the resultant supernatant was loaded onto a HisTrap HP column $(5 \mathrm{~mL})$ equilibrated with buffer A. The target protein was eluted with buffer B $(20 \mathrm{mM}$ sodium phosphate, $500 \mathrm{mM}$ sodium chloride, and $500 \mathrm{mM}$ imidazole, $\mathrm{pH} 7.4$ ), analyzed by $12.5 \%$ sodium dodecyl sulfate-polyacrylamide gel electrophoresis (SDS-PAGE), and quantified by the Bradford protein assay kit (Sangon, China).

To determine the native molecular weight of LhgR, gel-filtration chromatography was performed using a Superdex 200 10/300 GL column (GE Healthcare, USA) and standard proteins including thyroglobulin $(669 \mathrm{kDa})$, ferritin $(440 \mathrm{kDa})$, aldolase $(158 \mathrm{kDa})$, conalbumin $(75 \mathrm{kDa})$, ovalbumin $(43 \mathrm{kDa})$, and ribonuclease A $(13.7 \mathrm{kDa})$. The eluent buffer contained $50 \mathrm{mM}$ sodium phosphate and $150 \mathrm{mM}$ sodium chloride ( $\mathrm{pH} 7.2)$.

Electrophoretic mobility shift assays. Electrophoretic mobility shift assays (EMSAs) were carried out using the DNA fragment (F1 or F2) and purified LhgR The DNA fragments were first amplified by primer pairs F1-F/F1-R and F2-F/F2-R, respectively. Then, either fragment at a concentration of $10 \mathrm{nM}$ DNA was incubated with LhgR $(0-160 \mathrm{nM})$ in $20 \mu \mathrm{L}$ EMSA binding buffer $(10 \mathrm{mM}$ Tris- $\mathrm{HCl}$ [pH 7.4], $50 \mathrm{mM} \mathrm{KCl}, 0.5 \mathrm{mM}$ EDTA, $10 \%$ [vol/vol] glycerol, and $1 \mathrm{mM}$ dithiothreitol [DTT]). The binding reactions were carried out at $30^{\circ} \mathrm{C}$ for $30 \mathrm{~min}$. Electrophoresis was performed on $6 \%$ native polyacrylamide gels at $4{ }^{\circ} \mathrm{C}$ and $170 \mathrm{~V}$ (constant voltage) for about $45 \mathrm{~min}$, followed by staining with SYBR green I (TaKaRa, China) and photographing. Analysis of the interaction between $l h g O$ promoter region (F1) and LHGFR was performed using the same procedure.

To characterize the effector of LhgR, purified LhgR was first incubated with Llysine, 5-aminovalerate, glutarate, $\mathrm{L}-2-\mathrm{HG}, \mathrm{D}-2-\mathrm{HG}, 2-\mathrm{KG}$, or succinate at $30^{\circ} \mathrm{C}$ for $15 \mathrm{~min}$, followed by incubation with the added DNA fragments at $30^{\circ} \mathrm{C}$ for $30 \mathrm{~min}$. The mixtures were subsequently subjected to electrophoresis. 
DNase I footprinting. DNase I footprinting assays were performed using the 6carboxyfluorescein (FAM) labeled probe and purified LhgR. The DNA fragment F1 was PCR amplified using the primer pair F1-F/F1-R. The PCR products were cloned into the pEASY-Blunt plasmid using pEASY-Blunt Cloning Kit (TransGen, China). The FAM-labeled probes were PCR amplified using the resulting plasmid and the primer pair M13F-FAM/M13R. Then, $350 \mathrm{ng}$ probes were incubated with $2 \mu \mathrm{g}$ purified LhgR in a total volume of $40 \mu \mathrm{L}$ for $30 \mathrm{~min}$ at $30^{\circ} \mathrm{C}$. The DNase I digestion reaction was carried out by adding a total volume of $10 \mu \mathrm{L}$ solution containing approximately 0.015 units of DNase I (Promega, USA) and $100 \mathrm{nmol}$ $\mathrm{CaCl}_{2}$ and further incubating for $1 \mathrm{~min}$ at $37^{\circ} \mathrm{C}$, then stopped by adding a total volume of $140 \mu \mathrm{L}$ stop solution containing $0.15 \%$ (wt/vol) SDS, $200 \mathrm{mM}$ unbuffered sodium acetate, and $30 \mathrm{mM}$ EDTA. The digested DNA fragments were first extracted with phenol-chloroform, then precipitated with ethanol and resuspended in $30 \mu \mathrm{L}$ MiliQ water. The binding region of LhgR to DNA fragment F2 was analyzed using the same procedure.

Construction and purification of LHGFR. The genes encoding mTFP and Venus were synthesized by Tongyong Biosystem Co., Ltd (China). The mTFP gene and Venus gene were amplified and cloned into pETDuet-1 plasmid using the BamHI and SacI restriction sites, and SalI and NotI restriction sites, respectively. Then either the full-length $\ln g R$ gene, its truncated variants, or variants with artificial linkers were inserted between mTFP and Venus by the T5 exonuclease DNA assembly (TEDA) method ${ }^{52}$, respectively. The $\mathrm{L}-2-\mathrm{HG}$ biosensor LHGFR and its derivatives were expressed and purified using the same procedure. For expression in HEK293FT cells, the codon-optimized LHGFR ${ }_{0 \mathrm{~N} 3 \mathrm{C}}$, $\mathrm{LHGFR}_{0 \mathrm{~N} 7 \mathrm{C}}$, or LHGFR $_{3 \mathrm{~N} 7 \mathrm{C}}$ sequence was synthesized and cloned into pcDNA3.1 ${ }^{(+)}$plasmid behind a Kozak sequence, $5^{\prime}$-GCCACC- $3^{\prime}$. To construct the plasmid for mitochondrial expression of LHGFR, the gene of mitochondrial targeting sequence (MLSLRQSIRFFKPATRTLCSSRYLL) was PCR amplified using primer pair MitoF/Mito-R, and primer pair Mito-LHGFR-F/Mito-LHGFR-R was used to amplify the LHGFR fragment. The products were assembled using overlap PCR with MitoF/Mito-LHGFR-R, and cloned into pcDNA3.1 ${ }^{(+)}$plasmid behind a Kozak sequence using the NheI and NotI restriction sites.

Characterization of LHGFR in vitro. Purified L-2-HG biosensors and different compounds were diluted by $50 \mathrm{mM}$ Tris- $\mathrm{HCl}$ buffer ( $\mathrm{pH}$ 7.4), mixed together in a black 96-well plate at a volume ratio of 3:1, and the fluorescence intensities were measured using an EnSight microplate reader (PerkinElmer, USA) with excitation at $430 \mathrm{~nm}$, emission at $485 \mathrm{~nm}$ (mTFP) and $528 \mathrm{~nm}$ (Venus). The dose-response curves were fitted by OriginPro 2016 software (OriginLab) according to the following formula:

$$
R=R_{\max }+\frac{R_{\min }-R_{\max }}{1+\left([\mathrm{L}-2-\mathrm{HG}] / K_{d}\right)^{p}}
$$

where $R, R_{\min }$, and $R_{\max }$ refer to the emission ratio of Venus to mTFP, ratio in the absence of $\mathrm{L}-2-\mathrm{HG}$, and ratio at saturation with $\mathrm{L}-2-\mathrm{HG}$, respectively. The [L-2$\mathrm{HG}], K_{d}$, and $p$ refer to the $\mathrm{L}-2-\mathrm{HG}$ concentration, apparent dissociation constant, and Hill slope, respectively. Emission spectra were recorded at $430 \mathrm{~nm}$ excitation, in steps of $2 \mathrm{~nm}$. Excitation spectra were recorded using emission at $550 \mathrm{~nm}$, excitation from 380 to $535 \mathrm{~nm}$ in steps of $2 \mathrm{~nm}$.

Kinetics of L-2-HG binding by LHGFR were assessed by using SX-20 stoppedflow fluorimeter (Applied Photophysics, UK). Equal volumes of $1 \mu \mathrm{M}$ purified LHGFR in $50 \mathrm{mM}$ Tris-HCl buffer (pH 7.4) and L-2-HG-containing buffer (varying concentrations) were mixed, with data detected every four milliseconds at $430 \mathrm{~nm}$ excitation. Emission was detected by using a photomultiplier and a $515 \mathrm{~nm}$ longpass filter, and the detector voltage was set to $400 \mathrm{~V}$. Apparent rate constants ( $k^{\text {app }}$ $=k_{\text {on }}[\mathrm{L}-2-\mathrm{HG}]+k_{\text {off }}$ ) determined by fitting the Venus fluorescence increase after L-2-HG addition with a single exponential equation were plotted against L-2-HG concentrations ([L-2-HG]). Effects of temperature on LHGFR were detected by analyzing the dose-response curves for $\mathrm{L}-2-\mathrm{HG}$ at $25,28,31,34,37,40$, and $45^{\circ} \mathrm{C}$, respectively. The reversibility of LHGFR was determined by recording the emission ratios every minute after the addition of $5 \mu \mathrm{M}$ purified LhgO, the control test without the addition of $\mathrm{L}-2-\mathrm{HG}$ or purified LhgO was run in parallel. The $\mathrm{pH}$ stability of LHGFR was determined using $50 \mathrm{mM}$ Tris- $\mathrm{HCl}$ buffer with $\mathrm{pH}$ adjusted from 4.0 to 9.0. The background fluorescence without the addition of LHGFR was subtracted.

In order to test the ability of LHGFR for quantitative analysis L-2-HG in different biological samples, purified LHGFR was diluted by $50 \mathrm{mM}$ Tris- $\mathrm{HCl}(\mathrm{pH}$ 7.4), while varying concentrations of $\mathrm{L}-2-\mathrm{HG}$ were added into the serum and urine of a healthy adult and bacteria culture medium and filtered through a $0.22 \mu \mathrm{m}$ filter, respectively. The serum and urine were collected from a healthy adult (the first author of this article). The blood was collected by using promoting coagulating tubes and venous blood collection method, placed for $2 \mathrm{~h}$ at room temperature, and serum was prepared by centrifugation for $10 \mathrm{~min}$ at $2000 \times g$ and $4^{\circ} \mathrm{C}$. The urine collected from the experiment operator was diluted with an equal volume of $50 \mathrm{mM}$ Tris- $\mathrm{HCl}$ buffer ( $\mathrm{pH}$ 7.4). The processed serum and urine were filtered through a $0.22 \mu \mathrm{m}$ filter and stored at $-20^{\circ} \mathrm{C}$ until L-2-HG addition. The mixtures of purified LHGFR and L-2-HG in different biological samples were then incubated in a black 96-well plate at a volume ratio of 3:1, and the emission ratios were determined using an EnSight microplate reader. The background fluorescence without the addition of LHGFR was subtracted. The formulas for the quantitative analysis of $\mathrm{L}-2-\mathrm{HG}$ in different biological samples by $\mathrm{LHGFR}_{0 \mathrm{~N} 3 \mathrm{C}}$ and $\mathrm{LHGFR}_{0 \mathrm{~N} 7 \mathrm{C}}$ were as follows:

$$
[\mathrm{L}-2-\mathrm{HG}](\mu \mathrm{M})=26.20974 \times\left(\frac{0.79781}{2.17827-R}-1\right)^{1.1705}
$$

where $R$ refers to the emission ratio of Venus to mTFP determined by LHGFR $_{0 \mathrm{~N} 3 \mathrm{C}}$, and

$$
[\mathrm{L}-2-\mathrm{HG}](\mu \mathrm{M})=7.71913 \times\left(\frac{0.99281}{2.67083-R}-1\right)^{1.0787}
$$

where $R$ refers to the emission ratio of Venus to mTFP determined by LHGFR $_{0 \mathrm{~N} 7 \mathrm{C}}$

Quantification of L-2-HG by HPLC and LC-MS/MS. When L-2-HG was used as a carbon source to cultivate $P$. putida KT2440, its consumption was analyzed by using high-performance liquid chromatography (HPLC) system (Agilent 1100 series, Agilent Technologies, USA) equipped with an Aminex HPX-87H column $\left(300 \times 7.8 \mathrm{~mm}\right.$, Bio-Rad, USA) and a RID detector at $55^{\circ} \mathrm{C}$. The mobile phase was $0.1 \%$ formic acid at a flow rate of $0.4 \mathrm{~mL} \mathrm{~min}^{-1}$.

To detect L-2-HG concentrations in various biological samples by liquid chromatography-tandem mass spectrometry (LC-MS/MS) system, the samples containing D,L-2-hydroxyglutarate disodium salt (2,3,3-D3) as internal standard (ITSD) were centrifuged at $13,000 \times g$ for $15 \mathrm{~min}$, then filtered through a $0.22 \mu \mathrm{m}$ filter. The serum samples were mixed with methanol at a volume ratio of 1:3 and vortexed for $2 \mathrm{~min}$ to remove protein before centrifugation. Samples were analyzed by using a Thermo ultimate 3000 rapid separation liquid chromatography system (ThermoFisher, USA) coupled with a Bruker impact HD ESI-Q-TOF mass spectrometer (Bruker Daltonics, Germany) in negative ion mode and equipped with a Chirobiotic R column $(250 \times 4.6 \mathrm{~mm}$, Supelco Analytical, USA). The mobile phase was prepared from (A) $0.1 \%$ triethylamine adjusted to $\mathrm{pH} 4.5$ with acetic acid or (B) methanol. The quantification was conducted with an injection volume of $20 \mu \mathrm{L}$, a constant $5 \%$ gradient of (B) at a flow rate of $0.5 \mathrm{~mL} \mathrm{~min}^{-1}$, and a total analysis time of $15 \mathrm{~min}$.

Characterization of LHGFR in living bacteria. E. coli BL21(DE3) strains harboring either pETDuet-LHGFR ${ }_{0 N 3 C}$, pETDuet-LHGFR 0 LN7C, or pETDuet$\mathrm{LHGFR}_{3 \mathrm{~N} 7 \mathrm{C}}$ were grown to an $\mathrm{OD}_{600}$ of 0.6 in $\mathrm{LB}$ medium at $37^{\circ} \mathrm{C}$, after which the cells were induced overnight in the presence of $1 \mathrm{mM} \mathrm{IPTG}$ at $16^{\circ} \mathrm{C}$. The cultures were collected by centrifugation at $6000 \times g$ for $5 \mathrm{~min}$, washed three times, and resuspended to an $\mathrm{OD}_{600}$ of 2.5 by carbon starvation medium (MSM containing no carbon source) or glucose medium (MSM containing $20 \mathrm{mM}$ glucose).

To characterize the sensitivity and specificity of LHGFR expressed in $E$. coli BL21(DE3), $90 \mu \mathrm{L}$ cell suspensions following $8 \mathrm{~h}$ carbon starvation were mixed with $10 \mu \mathrm{L}$ increasing concentrations of $\mathrm{L}-2-\mathrm{HG}$ or other compounds, and then added into a black 96-well plate (total $100 \mu \mathrm{L} /$ per well), the fluorescence intensities were determined using an EnSight microplate reader (PerkinElmer, USA) and the following instrument settings: excitation at $430 \mathrm{~nm}$, emission at $485 \mathrm{~nm}$ (mTFP) and $528 \mathrm{~nm}$ (Venus), time intervals of $5 \mathrm{~min}$, the temperature at $37^{\circ} \mathrm{C}$, and shake at $180 \mathrm{rpm}$. For carbon starvation experiments, cell suspensions in carbon starvation medium or glucose medium were added into a black 96 -well plate $(100 \mu \mathrm{L} / \mathrm{per}$ well), then the fluorescence intensities were monitored every ten minutes. In order to analyze functions of CsiD and LhgO in endogenous L-2-HG anabolism and catabolism during carbon starvation, pETDuet-LHGFR ${ }_{0 N 3 C}$, pETDuetLHGFR $_{0 \mathrm{~N} 7 \mathrm{C}}$, or pETDuet-LHGFR ${ }_{3 \mathrm{~N} 7 \mathrm{C}}$ was transferred into E. coli MG1655(DE3) and its variants, and the assays were performed using the same procedure.

Cell culture and live-cell imaging. HEK293FT cells were cultured in high-glucose Dulbecco's modified eagle medium (DMEM) supplemented with $10 \%$ (vol/vol) fetal bovine serum (FBS), 100 units $\mathrm{mL}^{-1}$ penicillin, and $100 \mu \mathrm{gL}^{-1}$ streptomycin (all purchased from ThermoFisher, USA), and kept at $37^{\circ} \mathrm{C}$ in humidified air containing $5 \% \mathrm{CO}_{2}$. For hypoxia experiments, cells were kept in a compact $\mathrm{O}_{2}$ and $\mathrm{CO}_{2}$ subchamber controller (ProOx C21, BioSpherix, USA) at $2 \% \mathrm{O}_{2}, 5 \% \mathrm{CO}_{2}$ and balanced with $\mathrm{N}_{2}$ for $24 \mathrm{~h}$. For the construction of LHGFR expressing cell, HEK293FT cells were plated in 24-well plates so that they reached $70-90 \%$ confluency $24 \mathrm{~h}$ after plating, the medium was refreshed $2 \mathrm{~h}$ before transfection. Lipoplexes were first prepared in $50 \mu \mathrm{L}$ Opti-MEM Reduced Serum Medium (ThermoFisher, USA) per well containing $1.5 \mu \mathrm{L}$ Lipofectamine 3000 (ThermoFisher, USA) and $1 \mu \mathrm{g} \mathrm{pcDNA3.1}{ }^{(+)}$plasmid encoding either LHGFR $_{0 \mathrm{~N} 3 \mathrm{C}}$, LHGFR $_{0 \mathrm{NZC}}$, or LHGFR $3 \mathrm{N7C}$, and incubated for $15 \mathrm{~min}$ at room temperature, then added into the cell cultures.

For live-cell imaging, HEK293FT cells were plated on a poly-L-lysine pre-coated $35 \mathrm{~mm}$ glass-bottom dish and transfected with LHGFR after $24 \mathrm{~h}$. Live-cell imaging was carried out $48 \mathrm{~h}$ following transfection by using a Zeiss 880 confocal microscope equipped with an Objective C-Apochromat $\times 40 / 1.2 \mathrm{~W}$ autocorr M27 lens, a $458 \mathrm{~nm}$ argon laser, and a full-spectrum fluorescence detector. The emission of LHGFR expressed in HEK293FT cells was divided into a $463-495 \mathrm{~nm}$ channel (mTFP) and a 505-700 channel (Venus). Images were captured using 800 gain, $1024 \times 1024$ frame size, and 8 bit depth. The fluorescence intensities of each channel were analyzed in ZEN 3.1 software, and raw data were exported to Image- 
Pro Plus software for ratio image analysis. The Venus/mTFP emission ratio was calculated by dividing pixel-by-pixel a Venus image with a mTFP image. To realtime monitor the emission ratio of LHGFR in single living cells, single-cell with moderately fluorescent was imaged every 30 seconds, and $10 \mathrm{mM}$ L-2-HG was added into the cultures when the ratio of two-channel fluorescence intensities was stable.

Characterization of LHGFR in HEK293FT cells. To characterize the sensitivity and specificity of LHGFR expressed in HEK293FT cells, cells were trypsinized $48 \mathrm{~h}$ following transfection and suspended in $1 \times$ Hank's balanced salt solution supplemented with $20 \mathrm{mM}$ HEPES. Increasing concentrations of L-2-HG or other compounds including glutarate, D-2-HG, and glucose was mixed with the cell suspensions in a 96-well plate, respectively. Digitonin at a concentration of $10 \mu \mathrm{M}$ was used to induce cell permeabilization and deplete intracellular L-2-HG for in vivo response curves construction. Then, the fluorescence intensities were determined by a SpectraMax i3 fluorescence plate reader (Molecular Devices, USA) with excitation at $430 \mathrm{~nm}$ and emission at $485 \mathrm{~nm}$ (mTFP) and $528 \mathrm{~nm}$ (Venus). Basal L-2-HG concentration in HEK293FT cells under physiological conditions was determined by substituting the emission ratios of non-permeabilized HEK293FT cells into the calibrated in vivo response curves.

For the detection of hypoxia-induced production of $\mathrm{L}-2-\mathrm{HG}, \mathrm{LHGFR}_{0 \mathrm{~N} 3 \mathrm{C}}$ or LHGFR $_{3 \mathrm{~N} 7 \mathrm{C}}$ was expressed in HEK293FT cells and cultured sequentially under normoxic conditions for $24 \mathrm{~h}$ and hypoxic conditions for $24 \mathrm{~h}$ in the absence or presence of $5 \mathrm{mM}$ dimethyl-2-ketoglutarate (DMaKG). The preparation of cell suspensions and the measurement of emission ratios were performed using the same procedure. The background fluorescence was subtracted at each emission wavelength.

siRNA experiments. The following Silencer Select siRNAs used in this study were purchased from ThermoFisher Scientific (USA): negative control (4390846), L2HGDH-a (s36692), L2HGDH-b (s36693), LDHA (s351), and MDH2 (s8622). To analyze L2HGDH functions in L-2-HG catabolism, siRNA targeting L2HGDH and pcDNA3.1 $1^{(+)}$plasmid encoding either $\mathrm{LHGFR}_{0 \mathrm{~N} 3 \mathrm{C}}, \mathrm{LHGFR}_{0 \mathrm{~N} 7 \mathrm{C}}$, or LHGFR 3 N7C were mixed with Lipofectamine 3000 Transfection Reagent (ThermoFisher, USA) in Opti-MEM Reduced Serum Medium, and the lipoplexes prepared were transfected into HEK293FT cells according to the manufacturer's protocol. The fluorescence intensities were measured by a SpectraMax i3 fluorescence plate reader 48 h following transfection. Similarly, HEK293FT cells were transfected by LHGFR $_{0 \mathrm{~N} 3 \mathrm{C}}$ or LHGFR $3 \mathrm{~N}_{\mathrm{NC}}$ and siRNAs targeting LDHA and MDH2 separately or in combination. After transfection, cells were cultured sequentially under normoxic conditions for $24 \mathrm{~h}$ and hypoxic conditions for $24 \mathrm{~h}$ in the presence of $5 \mathrm{mM}$ $\mathrm{DMaKG}$, then the fluorescence intensities were measured. The cells cultured under the normoxic conditions in the presence of $5 \mathrm{mM}$ DMaKG for $48 \mathrm{~h}$ were set as control. The background fluorescence was subtracted at each emission wavelength.

Statistics and reproducibility. Software for initial data processing was Microsoft Excel 2016, and subsequent analyses were carried out using OriginPro 2016 (OriginLab), OriginPro 2019 (OriginLab), Graphpad Prism 5 (Graphpad), and Graphpad Prism 7 (Graphpad). The fluorescence intensities were determined by using Kaleido 3.0 (PerkinElmer) and SoftMax Pro software 7.0.2 (Molecular Devices). The imaging data were obtained and processed by Zen 3.1 (Zeiss) and Image-Pro Plus 6.0. All data shown are means \pm s.d. and were analyzed using oneway ANOVA test with Tukey's Multiple Comparison Test or two-tailed $t$-test where appropriate; ${ }^{*} P<0.05 ;{ }^{* *} P<0.01 ; * * * P<0.001 ;$ ns, no significant difference $(P \geq 0.05)$. For SDS-PAGE analyses, EMSAs, and fluorescence imaging experiments, similar results were obtained from three independent experiments. Detailed data analyses are described in the text.

Reporting summary. Further information on research design is available in the Nature Research Reporting Summary linked to this article.

\section{Data availability}

The data supporting the findings of this study are available within the article, its Supplementary Information files, and the Source Data file provided with this paper. A reporting summary for this article is available as a Supplementary Information file. Source data are provided with this paper.

Received: 23 March 2021; Accepted: 13 May 2021; Published online: 15 June 2021

\section{References}

1. Linster, C. L., Van Schaftingen, E. \& Hanson, A. D. Metabolite damage and its repair or pre-emption. Nat. Chem. Biol. 9, 72-80 (2013).
2. Intlekofer, A. M. et al. Hypoxia induces production of L-2-hydroxyglutarate. Cell Metab. 22, 304-311 (2015).

3. Oldham, W. M., Clish, C. B., Yang, Y. \& Loscalzo, J. Hypoxia-mediated increases in L-2-hydroxyglutarate coordinate the metabolic response to reductive stress. Cell Metab. 22, 291-303 (2015).

4. Harris, A. L. A new hydroxy metabolite of 2-oxoglutarate regulates metabolism in hypoxia. Cell Metab. 22, 198-200 (2015).

5. Hüdig, M. et al. Plants possess a cyclic mitochondrial metabolic pathway similar to the mammalian metabolic repair mechanism involving malate dehydrogenase and L-2-hydroxyglutarate dehydrogenase. Plant Cell Physiol. 56, 1820-1830 (2015).

6. Knorr, S. et al. Widespread bacterial lysine degradation proceeding via glutarate and L-2-hydroxyglutarate. Nat. Commun. 9, 5071 (2018).

7. Zhang, M. et al. Increased glutarate production by blocking the glutaryl-CoA dehydrogenation pathway and a catabolic pathway involving L-2hydroxyglutarate. Nat. Commun. 9, 2114 (2018).

8. Thompson, M. G. et al. Massively parallel fitness profiling reveals multiple novel enzymes in Pseudomonas putida lysine metabolism. mBio 10, e02577-18 (2019).

9. Rzem, R. et al. A gene encoding a putative FAD-dependent L-2hydroxyglutarate dehydrogenase is mutated in L-2-hydroxyglutaric aciduria. Proc. Natl Acad. Sci. USA 101, 16849-16854 (2004).

10. $\mathrm{Xu}, \mathrm{W}$. et al. Oncometabolite 2-hydroxyglutarate is a competitive inhibitor of a-ketoglutarate-dependent dioxygenases. Cancer Cell 19, 17-30 (2011).

11. Chowdhury, R. et al. The oncometabolite 2-hydroxyglutarate inhibits histone lysine demethylases. EMBO Rep. 12, 463-469 (2011).

12. Shim, E. H. et al. L-2-Hydroxyglutarate: an epigenetic modifier and putative oncometabolite in renal cancer. Cancer Discov. 4, 1290-1298 (2014).

13. Ye, D., Guan, K. L. \& Xiong, Y. Metabolism, activity, and targeting of D- and L2-hydroxyglutarates. Trends Cancer 4, 151-165 (2018).

14. Shelar, S. et al. Biochemical and epigenetic insights into L-2-hydroxyglutarate, a potential therapeutic target in renal cancer. Clin. Cancer Res. 24, 6433-6446 (2018).

15. Wang, J. H. et al. Prognostic significance of 2-hydroxyglutarate levels in acute myeloid leukemia in China. Proc. Natl Acad. Sci. USA 110, 17017-17022 (2013).

16. Tyrakis, P. A. et al. S-2-hydroxyglutarate regulates $\mathrm{CD} 8^{+}$T-lymphocyte fate Nature 540, 236-241 (2016)

17. Li, H. et al. Drosophila larvae synthesize the putative oncometabolite L-2hydroxyglutarate during normal developmental growth. Proc. Natl Acad. Sci. USA 114, 1353-1358 (2017).

18. Struys, E. A., Jansen, E. E., Verhoeven, N. M. \& Jakobs, C. Measurement of urinary D- and L-2-hydroxyglutarate enantiomers by stable-isotopedilution liquid chromatography-tandem mass spectrometry after derivatization with diacetyl-L-tartaric anhydride. Clin. Chem. 50, 1391-1395 (2004).

19. Calderón, C., Horak, J. \& Lämmerhofer, M. Chiral separation of 2hydroxyglutaric acid on cinchonan carbamate based weak chiral anion exchangers by high-performance liquid chromatography. J. Chromatogr. A 1467, 239-245 (2016).

20. Fernández-Galán, E. et al. Validation of a routine gas chromatography mass spectrometry method for 2-hydroxyglutarate quantification in human serum as a screening tool for detection of $i d h$ mutations. J. Chromatogr. B 1083, 28-34 (2018).

21. Strain, S. K., Groves, M. D., Olino, K. L. \& Emmett, M. R. Measurement of 2hydroxyglutarate enantiomers in serum by chiral gas chromatography -tandem mass spectrometry and its application as a biomarker for IDH mutant gliomas. Clin. Mass Spectrom. 15, 16-24 (2020).

22. Wang, $\mathrm{S}$. et al. Potassium channel selectivity filter dynamics revealed by single-molecule FRET. Nat. Chem. Biol. 15, 377-383 (2019).

23. Bischof, H. et al. Novel genetically encoded fluorescent probes enable realtime detection of potassium in vitro and in vivo. Nat. Commun. 8, 1422 (2017).

24. Zhang, W. H. et al. Monitoring hippocampal glycine with the computationally designed optical sensor GlyFS. Nat. Chem. Biol. 14, 861-869 (2018).

25. Surdo, N. C. et al. FRET biosensor uncovers cAMP nano-domains at $\beta$ adrenergic targets that dictate precise tuning of cardiac contractility. Nat. Commun. 8, 15031 (2017).

26. Mukherjee, S. et al. A novel biosensor to study cAMP dynamics in cilia and flagella. eLife 5, e14052 (2016).

27. Zhang, M. et al. Regulation of glutarate catabolism by GntR family regulator CsiR and LysR family regulator GcdR in Pseudomonas putida KT2440. mBio 10, e01570-19 (2019).

28. Thompson, M. G. et al. Robust characterization of two distinct glutarate sensing transcription factors of Pseudomonas putida $\mathrm{L}$-lysine metabolism. ACS Synth. Biol. 8, 2385-2396 (2019).

29. Rigali, S., Derouaux, A., Giannotta, F. \& Dusart, J. Subdivision of the helixturn-helix GntR family of bacterial regulators in the FadR, HutC, MocR, and YtrA subfamilies. J. Biol. Chem. 277, 12507-12515 (2002). 
30. Day, R. N., Booker, C. F. \& Periasamy, A. Characterization of an improved donor fluorescent protein for Förster resonance energy transfer microscopy. $J$. Biomed. Opt. 13, 031203 (2008).

31. Nagai, T. et al. A variant of yellow fluorescent protein with fast and efficient maturation for cell-biological applications. Nat. Biotechnol. 20, 87-90 (2002).

32. Hires, S. A., Zhu, Y. \& Tsien, R. Y. Optical measurement of synaptic glutamate spillover and reuptake by linker optimized glutamate-sensitive fluorescent reporters. Proc. Natl Acad. Sci. USA 105, 4411-4416 (2008).

33. Chen, X., Zaro, J. L. \& Shen, W. C. Fusion protein linkers: property, design and functionality. Adv. Drug Deliv. Rev. 65, 1357-1369 (2013).

34. Steffen, V. et al. A toolbox of genetically encoded FRET-based biosensors for rapid L-lysine analysis. Sensors 16, 1604 (2016).

35. Yaginuma, H. et al. Diversity in ATP concentrations in a single bacterial cell population revealed by quantitative single-cell imaging. Sci. Rep. 4, 6522 (2014).

36. Marschall, C. et al. Molecular analysis of the regulation of $c s i D$, a carbon starvation-inducible gene in Escherichia coli that is exclusively dependent on $\sigma^{S}$ and requires activation by cAMP-CRP. J. Mol. Biol. 276, 339-353 (1998).

37. Metzner, M., Germer, J. \& Hengge, R. Multiple stress signal integration in the regulation of the complex $\sigma^{\mathrm{S}}$-dependent csiD-ygaF-gabDTP operon in Escherichia coli. Mol. Microbiol. 51, 799-811 (2004).

38. Kranendijk, M., Struys, E. A., Salomons, G. S., Van der Knaap, M. S. \& Jakobs, C. Progress in understanding 2-hydroxyglutaric acidurias. J. Inherit. Metab. Dis. 35, 571-587 (2012).

39. Rakheja, D. et al. Papillary thyroid carcinoma shows elevated levels of 2hydroxyglutarate. Tumour Biol. 32, 325-333 (2011).

40. Liang, M. et al. A CRISPR-Cas12a-derived biosensing platform for the highly sensitive detection of diverse small molecules. Nat. Commun. 10, 3672 (2019).

41. Cao, J. et al. Harnessing a previously unidentified capability of bacterial allosteric transcription factors for sensing diverse small molecules in vitro. Sci. Adv. 4, eaau4602 (2018).

42. Grazon, C. et al. A progesterone biosensor derived from microbial screening. Nat. Commun. 11, 1276 (2020).

43. Moroni, I. et al. L-2-Hydroxyglutaric aciduria and brain malignant tumors: a predisposing condition? Neurology 62, 1882-1884 (2004).

44. Janin, M. et al. Serum 2-hydroxyglutarate production in IDH1- and IDH2mutated de novo acute myeloid leukemia: A study by the Acute Leukemia French Association group. J. Clin. Oncol. 32, 297-305 (2014).

45. Dang, L. et al. Cancer-associated IDH1 mutations produce 2hydroxyglutarate. Nature 462, 739-744 (2009).

46. Kalliri, E., Mulrooney, S. B. \& Hausinger, R. P. Identification of Escherichia coli $\mathrm{YgaF}$ as an L-2-hydroxyglutarate oxidase. J. Bacteriol. 190, 3793-3798 (2008).

47. Lobas, M. A. et al. A genetically encoded single-wavelength sensor for imaging cytosolic and cell surface ATP. Nat. Commun. 10, 711 (2019).

48. Jing, M. et al. A genetically encoded fluorescent acetylcholine indicator for in vitro and in vivo studies. Nat. Biotechnol. 36, 726-737 (2018).

49. Zhao, Y. et al. SoNar, a highly responsive $\mathrm{NAD}^{+} / \mathrm{NADH}$ sensor, allows highthroughput metabolic screening of anti-tumor agents. Cell Metab. 21, 777-789 (2015).

50. Yong, C., Stewart, G. D. \& Frezza, C. Oncometabolites in renal cancer. Nat. Rev. Nephrol. 16, 156-172 (2019).

51. Schäfer, A. et al. Small mobilizable multi-purpose cloning vectors derived from the Escherichia coli plasmids pK18 and pK19: selection of defined deletions in the chromosome of Corynebacterium glutamicum. Gene 145, 69-73 (1994)
52. Xia, Y. et al. T5 exonuclease-dependent assembly offers a low-cost method for efficient cloning and site-directed mutagenesis. Nucleic Acids Res. 47, e15 (2018).

\section{Acknowledgements}

This work was supported by the grants of the National Key R\&D Program of China (2018YFA0901200), National Natural Science Foundation of China (31970055), Shandong Provincial Funds for Distinguished Young Scientists (JQ 201806), and Qilu Young Scholar of Shandong University. The funders had no role in study design, data collection, and interpretation, or the decision to submit the work for publication. We also thank Dr. Zhifeng Li, Dr. Jingyao Qu, and Dr. Jing Zhu from SKLMT (State Key Laboratory of Microbial Technology, Shandong University) for assistance in the mass spectrographic analysis, Dr. Haiyan Yu, Dr. Yuyu Guo, and Dr. Xiaomin Zhao from SKLMT for assistance in microimaging of laser scanning confocal microscope analysis.

\section{Author contributions}

C.G., C.M., and P.X. designed the research. Z.K., M.Z., K.G., W.M., Y.L., D.X., W.Z., and S.G. performed the research. Z.K. and C.G. analyzed the data. Z.K., C.G., C.M., and P.X. wrote the paper.

\section{Competing interests}

The authors declare no competing interests.

\section{Additional information}

Supplementary information The online version contains supplementary material available at https://doi.org/10.1038/s41467-021-23723-7.

Correspondence and requests for materials should be addressed to C.G. or P.X.

Peer review information Nature Communications thanks Andrew Intlekofer and the other, anonymous, reviewer(s) for their contribution to the peer review of this work. Peer reviewer reports are available.

Reprints and permission information is available at http://www.nature.com/reprints

Publisher's note Springer Nature remains neutral with regard to jurisdictional claims in published maps and institutional affiliations.

(c) (i)

Open Access This article is licensed under a Creative Commons Attribution 4.0 International License, which permits use, sharing, adaptation, distribution and reproduction in any medium or format, as long as you give appropriate credit to the original author(s) and the source, provide a link to the Creative Commons license, and indicate if changes were made. The images or other third party material in this article are included in the article's Creative Commons license, unless indicated otherwise in a credit line to the material. If material is not included in the article's Creative Commons license and your intended use is not permitted by statutory regulation or exceeds the permitted use, you will need to obtain permission directly from the copyright holder. To view a copy of this license, visit http://creativecommons.org/ licenses/by/4.0/.

(C) The Author(s) 2021 